\title{
Multiple Applications of a Transient CRISPR-Cas9 Coupled with Electroporation (TRACE) System in the Cryptococcus neoformans Species Complex
}

\author{
Yumeng Fan and Xiaorong Lin ${ }^{1}$ \\ Department of Microbiology, University of Georgia, Athens, Georgia 30602 \\ ORCID IDs: 0000-0003-4975-3751 (Y.F.); 0000-0002-3390-8387 (X.L.)
}

\begin{abstract}
Cryptococcus neoformans is a fungal pathogen that claims hundreds of thousands of lives annually. Targeted genetic manipulation through biolistic transformation in C. neoformans drove the investigation of this clinically important pathogen at the molecular level. Although costly and inefficient, biolistic transformation remains the major method for editing the Cryptococcus genome as foreign DNAs introduced by other methods such as electroporation are predominantly not integrated into the genome. Although the majority of DNAs introduced by biolistic transformation are stably inherited, the transformation efficiency and the

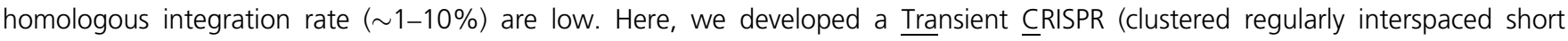
palindromic repeat)-Cas9 coupled with Electroporation (TRACE) system for targeted genetic manipulations in the $C$. neoformans species complex. This method took advantages of efficient genome integration due to double-strand breaks created at specific sites by the transient CRISPR-Cas9 system and the high transformation efficiency of electroporation. We demonstrated that TRACE can efficiently generate precise single-gene deletion mutants using the $A D E 2$ locus as an example. This system can also effectively delete multiple genes in a single transformation, as evident by the successful generation of quadruple $m f \alpha 1 \Delta 2 \Delta 3 \Delta 4 \Delta$ mutants. In addition to generating gene deletion mutants, we complemented the ade $2 \Delta$ mutant by integrating a wild-type ADE2 allele at the "safe haven" region (SH2) via homologous recombination using TRACE. Interestingly, introduced DNAs can be inserted at a designated genetic site without any homologous sequences, opening up numerous other applications. We expect that TRACE, an efficient, versatile, and costeffective gene editing approach, will greatly accelerate research in this field.
\end{abstract}

KEYWORDS Cryptococcus neoformans; electroporation; biolistic transformation; CRISPR-Cas9; gene disruption; gene complementation; ectopic integration; double-strand break; gene family

RYPTOCOCCUS neoformans is a ubiquitous environmental pathogen that claims hundreds of thousands of lives annually (Park et al. 2009; Perfect et al. 2010; Brown et al. 2012; Rothe et al. 2013; Armstrong-James et al. 2014; Chaiwarith et al. 2014; Gaskell et al. 2014; Idnurm and Lin 2015; Perfect and Bicanic 2015). The $C$. neoformans species complex contains serotype $A$, serotype $\mathrm{D}$, and the $\mathrm{AD}$ hybrid, with serotype A responsible for the vast majority of cryptococcosis

Copyright (c) 2018 by the Genetics Society of America doi: https://doi.org/10.1534/genetics.117.300656

Manuscript received December 20, 2017; accepted for publication February 7, 2018; published Early Online February 14, 2018.

Supplemental material is available online at www.genetics.org/lookup/suppl/doi:10. 1534/genetics.118.300656/-/DC1.

${ }^{1}$ Corresponding author: Department of Microbiology, University of Georgia, 206 Biological Sciences Building, 120 Cedar St., Athens, GA 30602. E-mail: xiaorong.lin@uga.edu cases (Casadevall and Perfect 1998; Lin and Heitman 2006). The past two decades have seen great progress in our understanding of cryptococcal biology and pathology, largely due to the ability to genetically modify this eukaryotic pathogen through targeted mutagenesis since the early 1990s (Edman and Kwon-Chung 1990; Toffaletti et al. 1993).

Electroporation was first reported in 1990 to generate gene deletion mutants in serotype D strains using auxotrophic selection markers (Edman and Kwon-Chung 1990). Although electroporation can yield hundreds to thousands of transformants per transformation, the vast majority of the transformants are unstable because of nonintegration of the introduced DNA (often $<0.1 \%$ transformants are stable) (Edman 1992; Varma et al. 1992; Lin et al. 2015). The adoption of split markers (Fu et al. 2006; Kim et al. 2009), the use of dominant drug selection markers (McDade and Cox 2001; 
Fox et al. 2003), and the employment of a recipient strain deficient in nonhomologous end joining (NHEJ) (Walker et al. 2001; Goins et al. 2006) increased the genome integration events among transformants generated by electroporation ( 10\%) (Lin et al. 2015). Nonetheless, electroporation remains an inefficient approach to make targeted genetic mutations due to the predominance of nonintegration of the introduced DNA and the predilection of ectopic DNA insertion even when genome integration does occur.

The introduction of biolistic transformation in 1993 was a watershed moment to this field (Toffaletti et al. 1993) because most transformants are stable, with introduced DNA integrated into the genome (Lodge et al. 1994; Kim et al. 2009). Biolistic transformation soon became THE method for genome editing in Cryptococcus. However, this method of choice is far from satisfactory. The number of transformants obtained by biolistic transformation varies, with typically 10-100 transformants in serotype A strains like H99 and 0-20 transformants in serotype D strains like JEC21 or XL280 in our setting. With low rates of homologous recombination (HR) (1-10\%) in the C. neoformans species complex, repeated transformations are often necessary to identify desired mutants. Moreover, biolistic transformation relies on the expensive biolistic PDS-1000/He Particle Delivery System that is only available from Bio-Rad (Hercules, CA; \$25,000 in 2017) and the procedure requires costly consumables such as gold beads. Thus, a cost-effective approach with higher transformation efficiency and higher frequency of HR is desired.

The low rate of homologous integration is a limiting factor in targeted genetic manipulation in Cryptococcus. NHEJ is preferred over HR during the nondivision phase (Sonoda et al. 2006; Arras et al. 2016). Compromising NHEJ (e.g., by deletion of the CKU80 gene) can enrich the HR events among transformants. However, relying on the CKU80 deletion mutant as the recipient strain is restricting and problematic given the potential role of Cku80 in infection and stress adaptation (Liu et al. 2008). Another approach to increase the $\mathrm{HR}$ rate is to create DNA double-strand breaks (DSBs) (Haber 2000). Interestingly, the clustered regularly interspaced short palindromic repeat (CRISPR) and CRISPR-associated gene 9 (CRISPR-Cas9) system from bacteria and archaea can also create DSBs as a defense mechanism (Barrangou et al. 2007; Bhaya et al. 2011; Ran et al. 2013). Two essential elements in the CRISPR-Cas9 system are the single-guide RNA (sgRNA) and the endonuclease Cas9. sgRNA carries a 20-nt sequence that can hybridize to the complementary DNA region and leads Cas9 to generate a DSB when a protospacer-adjacent motif (PAM) sequence immediately follows the target sequence. This feature makes CRISPR-Cas9 a powerful genome editing tool that has been applied in multiple eukaryotes (Hsu et al. 2014; Mali et al. 2013), including fungal pathogens (Nødvig et al. 2015) such as Candida albicans (Vyas et al. 2015; Min et al. 2016) and Aspergillus fumigatus (Fuller et al. 2015; Zhang et al. 2016). Two different CRISPR-Cas9 systems have been reported in $C$. neoformans (Arras et al. 2016; Wang et al. 2016) and both systems can generate single gene deletion mutants efficiently. Here, we developed a simpler approach named TRACE (Transient CRISPR-Cas9 coupled with Electroporation) that couples the highly efficient electroporation with a transiently expressed CRISPR-Cas9 system for multiple applications in targeted genetic manipulation in the $C$. neoformans species complex. A similar transiently expressed CRISPR-Cas9 system is widely adopted in the Candida field (Min et al. 2016). TRACE has become a method of choice in our laboratory to genetically modify $C$. neoformans. We hope that this approach will also greatly facilitate other cryptococcal researchers in their investigations.

\section{Materials and Methods}

\section{Strains and growth conditions}

Strains used and generated in this study are listed in Supplemental Material, Table S1 in File S1. Strains were stored at $-80^{\circ}$ as glycerol stocks. Freshly revived strains were maintained on YPD (1\% yeast extract, $2 \%$ BactoPeptone, and $2 \%$ dextrose) agar medium at $30^{\circ}$ unless indicated otherwise. Cryptococcus transformants were selected on YPD medium supplemented with NAT (nourseothricin, $100 \mu \mathrm{g} / \mathrm{ml}$ ) or G418 $(200 \mu \mathrm{g} / \mathrm{ml}$ ) for selection, as described previously (Lin et al. 2015).

\section{Genomic DNA extraction}

Genomic DNA of the reference wild-type strains H99, XL280, and JEC21 was extracted using a CTAB protocol as described previously (Pitkin et al. 1996). Genomic DNA of the Cryptococcus transformants examined in this study was extracted using a mini-preparation protocol. Briefly, cells were collected from overnight cultures on YPD plates and suspended in $500 \mu \mathrm{l}$ of lysis buffer (100 mM Tris pH 8.0, $50 \mathrm{mM}$ EDTA, and 1\% SDS). Next, 150-200 $\mu \mathrm{l} 0.5 \mathrm{~mm}$ disruption glass beads (RPI, catalog \#9831) were added to the cell suspension, and cells were then vortexed for $2 \mathrm{~min}$ followed by centrifugation at $16,000 \times g$ for $3 \mathrm{~min}$. The supernatant was mixed with $275 \mu \mathrm{l}$ of $7 \mathrm{M}$ ammonium acetate, incubated at $65^{\circ}$ for $5 \mathrm{~min}$, and then chilled on ice for 3-5 min. The samples were then treated with chloroform $(500 \mu \mathrm{l})$ twice and DNA in the upper layer was precipitated by isopropanol. The precipitated DNA samples were washed with 70\% ethanol, air dried, and dissolved in $100 \mu$ l sterile water.

\section{Construction of the Cas9, the sgRNA, and the gene deletion cassettes}

To generate a cryptococcal Cas9 expression construct, the Cas 9 coding sequence was amplified from the plasmid pDD162 (Addgene, MA) (Dickinson et al. 2013) by PCR. A FseI restriction enzyme site was added to the $5^{\prime}$ end of the Cas9 sequence and a PacI restriction enzyme site was added to the $3^{\prime}$ end using specifically designed primers (all primers used in this study are listed in Table S2 in File S1). The PCR product was purified using the Invitrogen Quick PCR purification kit (Carlsbad, CA) and digested with FseI and PacI restriction enzymes. The digested product was ligated into 
the plasmid pXL1 between the GPD1 promoter and the GPD1 terminator, as described previously (Xue et al. 2006; Wang et al. 2014). The resulting plasmid pXL1-Cas9 was used as the template to amplify the whole cryptococcal Cas9 expression cassette for transformation (Figure 1).

To generate the sgRNA construct with the desired target sequence, we first obtained the targeted cryptococcal gene sequences from FungiDB.org (Stajich et al. 2012). The genome sequences of the H99 and XL280 strains were obtained from the National Center for Biotechnology Information (PRJNA411 and PRJNA217913) (Ni et al. 2013; Janbon et al. 2014). The guiding sequence of sgRNA was designed using sgRNAcas9_V3.0_GUI software (Zhao et al. 2015) with the default parameters. The only exception was that the potential single-sgRNA target sequences were searched on both strands (sgRNA length: $20 \mathrm{nt}$; GC\%: 40 60\%; offset distance of paired-gRNAs: $-2 \sim 32$; and maximum number of mismatches: 5). The 20-nt selected guiding sequence was added to the primers. Cryptococcus native U6 promoter (Wang et al. 2016) was amplified from JEC21 while the scaffold of sgRNA was amplified from the plasmid pDD162. The U6 promoter, 20-nt guide sequence, scaffold, and 6-T terminator were assembled in the order shown in Figure 1 using a single-joint PCR as described previously (Min et al. 2016).

To generate the gene deletion construct, we first amplified the nourseothricin acetyltransferase gene NAT1 expression cassette (NAT) and neomycin resistance gene expression cassette (NEO) selection markers by PCR from the plasmids pPZPNATcc and pPZP-NEO1 using primers M13F and M13R (Walton et al. 2005). NAT and NEO confer Cryptococcus resistance to clonNAT and G418, respectively. Homologous arms ( $1 \mathrm{~kb}$ or $500 \mathrm{bp}$ ) of the targeted genes were amplified from the genome of the indicated strain background (H99, JEC21, or $\mathrm{XL} 280$ ). The $5^{\prime}$ and $3^{\prime}$ homologous arms and the drug marker were assembled as shown in Figure 1 by a double-joint PCR as described previously (Kim et al. 2009; Lin et al. 2015). For the gene deletion construct with only 50-bp homologous arms, the 50-bp homologous sequences were included in the designed primers as overhangs. The resulting primers were used to amplify the drug selection marker.

All three PCR products (the Cas9 cassette, the sgRNA cassette, and the gene knockout construct) were purified using an Invitrogen Quick PCR purification kit and eluted with water. Purified products were concentrated by speed vacuum when necessary. These products were then used in electroporation as described below.

\section{Electroporation in Cryptococcus}

Electroporation was performed as we described previously with some minor modifications (Edman and Kwon-Chung 1990; Lin et al. 2015). Briefly, Cryptococcus cells were streaked from glycerol stocks stored at $-80^{\circ}$ and cultured on YPD agar plates at $30^{\circ}$ before being transferred to $3 \mathrm{ml}$ of YPD liquid medium. Cells were grown overnight at $30^{\circ}$ with shaking at $250 \mathrm{rpm}$. The overnight culture was then transferred into fresh YPD medium $(100 \mathrm{ml})$ with the initial inoculum of
$\mathrm{OD}_{600}=0.2$. Cells were cultured for additional 4-5 hr until the cell density reached $\mathrm{OD}_{600}$ between 0.6 and 1.0. Cells were collected by centrifugation at $3200 \times g$ for $5 \mathrm{~min}$ at $4^{\circ}$. The pelleted cells were washed twice with ice-cold water before being suspended in $10 \mathrm{ml}$ ice-cold electroporation buffer (EB) buffer (10 mM Tris- $\mathrm{HCl} \mathrm{pH} \mathrm{7.5,} 1 \mathrm{mM} \mathrm{MgCl}_{2}$, and $270 \mathrm{mM}$ sucrose) with $1 \mathrm{mM}$ DTT. After $1 \mathrm{hr}$ of incubation on ice, cells were harvested by centrifugation and were resuspended in EB buffer $(250 \mu \mathrm{l})$. Cell suspension $(45 \mu \mathrm{l})$ was mixed gently with DNA ( $5 \mu \mathrm{l}$ ) in a precooled $2 \mathrm{~mm}$ gap electroporation cuvette. Electroporation was done using an Eppendorf multiporator with the bacterial mode $(\mathrm{V}=2 \mathrm{kV}$ with $\tau$ optimized for $5 \mathrm{msec}$ ). The electroporated cells were then suspended in $1 \mathrm{ml}$ of YPD medium and cultured at $30^{\circ}$ for $2 \mathrm{hr}$ before being plated onto the appropriate selective agar medium (YPD + NAT or YPD + G418). Transformants were counted after 2 days of incubation at $30^{\circ}$. For the screen of ade $2 \Delta$ mutants, transformants were kept at $4^{\circ}$ for an additional 2 days or more for pigment accumulation for visual examination.

\section{Stability testing of transformants}

Stability testing was performed as we described previously (Lin et al. 2015). Briefly, transformants were transferred to fresh selective agar medium (YPD + NAT or YPD + G418). After 2 days of incubation at $30^{\circ}$, plates were replicated onto nonselective YPD plates. After incubation at $30^{\circ}$ for $24 \mathrm{hr}$, cells were replicated again onto fresh nonselective YPD plates. At the fifth passage, cells were replicated onto nonselective YPD plates as well as appropriate selective drug plates. Colonies growing on the selective drug plates were compared to the corresponding ones on the nonselective YPD plates. Colonies with spotty growth on the selective medium compared to that on the nonselective medium were considered unstable transformants. Only colonies that showed similar growth on both selective and nonselective media were considered to be stable transformants.

\section{Diagnostic RFLP assay}

For the diagnostic RFLP assay screening for the ADE2 gene deletion, primers as indicated in Figure 3A-i (H99_ADE2_FLF and H99_ADE2_RR) were used to amplify the DNA fragment from the selected transformants and the control strains (wild-type and a known ade2 $\Delta$ mutant YP27 (Lin et al. 2015)). For strains in the XL280 background, wildtype should yield a band $\sim 4.4 \mathrm{~kb}$ in size while the correct gene deletion mutant should give a band of $3.6 \mathrm{~kb}$ in size. For strains in the H99 strain background, both wild-type and the correct $A D E 2$ gene deletion mutants should yield PCR amplicons of $\sim 4.2 \mathrm{~kb}$ in size. To distinguish them, the PCR products were purified and digested with the restriction enzyme NotI. Because a NotI cutting site is present inside the NAT marker, the digested PCR products of the correct gene deletion mutants should yield two bands of 2.3 and $1.8 \mathrm{~kb}$ in size, respectively, while wild-type should show only one $4.2 \mathrm{~kb}$ band. 

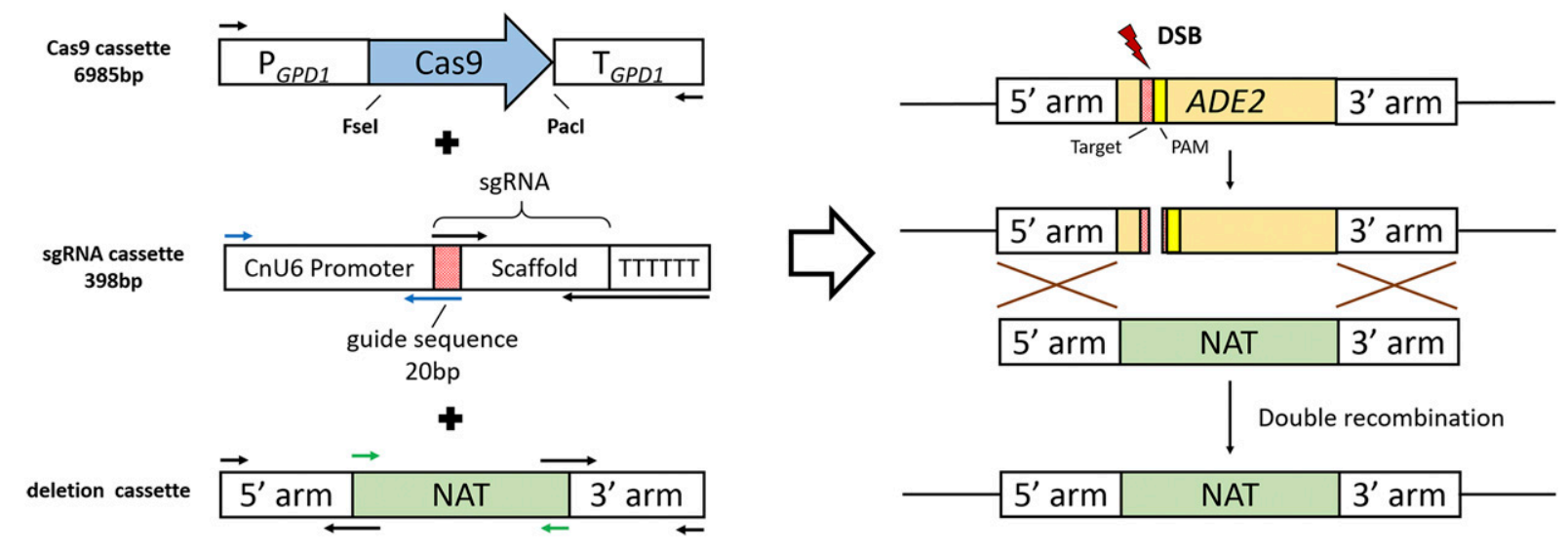

Figure 1 Construction of the Transient CRISPR (clustered regularly interspaced short palindromic repeat)-Cas9 coupled with Electroporation (TRACE) system. A diagram of the three elements of the TRACE system generated via PCR (left panel). Arrows represent the position and the direction of the primers. A diagram for the working concept of the TRACE system (right panel). When the three elements are all expressed in Cryptococcus cells, singleguide RNA (sgRNA) will guide Cas9 to the specific site that matches the target sequence and then Cas9 will generate a double-strand break (DSB). The deletion construct will serve as the template during DSB repair and eventually replace the gene of interest with the drug-resistant marker by homologous recombination. NAT: nourseothricin resistance cassette

\section{DNA sequencing}

DNA sequencing was used to examine the integration of the introduced DNA at the specific insertion site. For the ADE2 deletion, candidates generated from the deletion construct with only 50-bp homologous arms (detailed in the Results section and primers shown in Figure 4C) were used to amplify the potential inserted region. For the $A D E 2$ complementation candidates generated by insertion of the complementation construct with no homologous arms (detailed in the Results section), primers SH2_FLF and SH2_RR were used to amplify the potential inserted region. PCR products were purified and sent to Eurofins Genomics for Sanger Sequencing. Primers for sequencing are also listed in Table S2 in File S1.

\section{Mating assay}

Cells were cultured in YPD medium and then suspended in sterile water. The concentration of cells was adjusted to $\mathrm{OD}_{600}=3.0$. Equal volumes of cells of the mating pairs ( $\alpha$ and a strains as indicated in Figure 7E) were mixed and $4 \mu \mathrm{l}$ of the mating mixture was dropped onto $\mathrm{V} 8$ juice agar medium $(\mathrm{pH}=5)$. The cocultures were incubated in the dark at $22^{\circ}$ for 1 week and the colony images were taken with a GO-21 camera under an Olympus SZX16 stereoscope.

\section{Data availability}

Strains and plasmids are available upon request. The authors affirm that all data necessary for confirming the conclusions of the article are present within the article, figures, tables, and supplemental file.

\section{Results}

\section{Construction of the transient CRISPR-Cas9 coupled with electroporation (TRACE) system}

Two previous studies adopted different CRISPR-Cas9 systems in the transformation of $C$. neoformans. Increased homologous integration rates were observed in both studies (Arras et al. 2016; Wang et al. 2016). One study used a "suicide" CRISPR-Cas9 system, in which sgRNA and Cas9 cassettes $(>5 \mathrm{~kb})$ were added to one of the homologous arms of the deletion construct in a single vector. After HR upon transformation, the CRISPR-Cas9 elements will be eliminated without integrating into the genome (Wang et al. 2016). This method requires the construction of one complicated plasmid carrying all three elements and the deletion construct each time. The other study used a strain with stably integrated CAS9 as the recipient in the biolistic transformation (Arras et al. 2016). Thus, the drawbacks of biolistic transformation remain and the need to have recipients with integrated CAS9 in their genome is limiting. In C. albicans, a transiently expressed CRISPR-Cas9 system can generate gene deletion efficiently without stable integration of CAS9 (Min et al. 2016). Here, we decided to determine if the transiently expressed CRISPR-Cas9 system will enable efficient genetic manipulation in C. neoformans. The system consists of three independent components that can be generated by PCR: the Cas9 expression cassette, the sgRNA expression cassette, and the donor DNA (e.g., the gene deletion construct shown in the left panel of Figure 1).

For the Cas9 expression cassette, we first generated the plasmid pXL1-Cas9, in which the expression of the CAS9 gene was driven by the constitutively expressed GPD1 promoter from Cryptococcus. A primer upstream of the GPD1 promoter and a primer at the end of the GPD1 terminator were used to amplify the whole 7-kb construct from plasmid pXL1-Cas9 by PCR.

For the single guide RNA cassette, we chose the Cryptococcus U6 promoter to drive its expression as it has been used previously (Wang et al. 2016). The scaffold sequence of the sgRNA cassette was amplified from the plasmid pDD162 and six consecutive thymines (6-T) were included as the terminator. The 20-nt target sequence of sgRNA was designed to match the specific sequence in the Cryptococcus genome that needed to be edited. This sequence was added to primers as 
H99
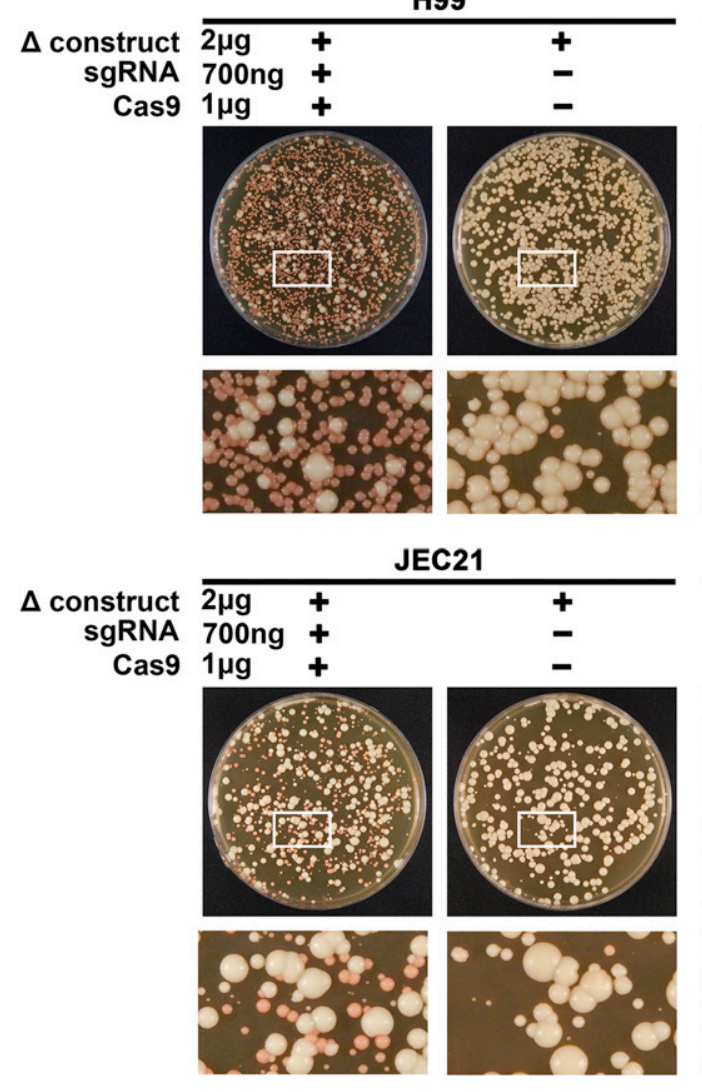

EC21

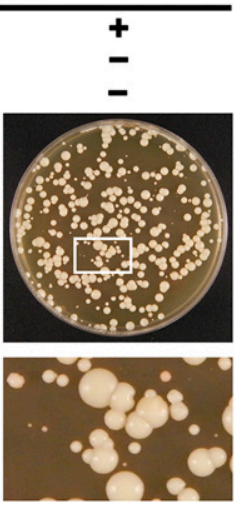

cku80வ H99

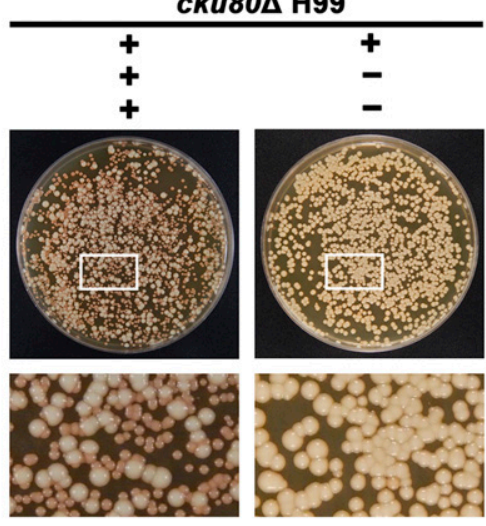

XL280

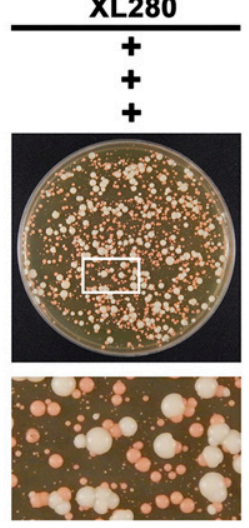

Figure 2 TRACE [Transient CRISPR (clustered regularly interspaced short palindromic repeat)-Cas9 coupled with Electroporation] can dramatically increase the gene disruption rate. The TRACE system was used to generate ADE2 deletion mutants in both serotype A (H99 and cku80 $\mathrm{H}$ H9) and serotype D (JEC21 and $\mathrm{XL280)}$ strains. Electroporation with no Cas9/ sgRNA (single-guide RNA) was done in parallel. The deletion construct was maintained at the same concentration. Red colonies indicate mutants with disrupted $A D E 2$. an overhang. The U6 promoter, the target sequence, and the scaffold sequence together with the 6-T terminator were assembled by single-joint PCR, as shown in Figure 1 .

For the donor DNA, we used a gene deletion construct. A gene complementation construct or constructs for other purposes could also be used (see the Materials and Methods section and the Results section "Complementation by ectopic insertion into the specific safe haven region does not need any homologous arm" for details). The gene deletion construct included a 1-kb 5' homologous arm, a drug selection marker, and a 1-kb 3' homologous arm (left panel of Figure 1). These three elements were assembled by double-joint PCR as described previously (Kim et al. 2009; Lin et al. 2015).

We planned to mix these three components and introduce them into Cryptococcus cells together. The transformants will be selected based on the marker used in the donor DNA. In theory, if all three fragments were successfully introduced and expressed in cryptococcal cells, the 20-nt target sequence of sgRNA would recognize and hybridize to a specific sequence in the Cryptococcus genome (e.g., the site in the ADE2 gene as depicted in the right panel of Figure 1). Cas9 would be recruited by the sgRNA and generate a DSB 3-bp upstream of the PAM sequence (Jinek et al. 2012; Ran et al. 2013). The donor DNA, in this case the gene deletion construct, would be used as the template to repair the DSB by HR, consequently replacing the gene with the drug-resistance marker (Figure 1, right panel).

To deliver these three DNA elements into the cells, we decided to use electroporation rather than biolistic bombardment because of the high transformation efficiency and low cost of the former approach. Although the predominance of nonintegration of introduced DNA has rendered electroporation an inferior method for genetic mutations in Cryptococcus, we hypothesize that the DSB created by Cas9 will drastically increase the DNA integration events. Thus, the coupling of electroporation with TRACE should make it a better method for targeted mutagenesis in C. neoformans.

\section{Generation of the ADE2 single-gene deletion mutants by TRACE}

To test the efficiency of the TRACE system in the $C$. neoformans species complex, we decided to use this system to delete the phosphoribosylaminoimidazole carboxylase $A D E 2$ gene in both serotype A and serotype D strains. The ade $2 \Delta$ mutant is known to accumulate red pigment and thus can be screened visually. We built the $A D E 2$ gene deletion constructs for both serotype A and D stains, which included an $\sim 1-\mathrm{kb} 5^{\prime}$ homologous arm and a 1-kb 3' homologous arm. We selected an sgRNA-targeting site 648-bp downstream of the translation start site (ATG) of $A D E 2$ in the serotype A reference strain H99. The DSB site would be $\sim 600$ bp away from the $5^{\prime}$ end and $1.2 \mathrm{~kb}$ away from $3^{\prime}$ end of the $A D E 2$ sequences being deleted. For the serotype D reference strains JEC21 and XL280, we selected the sgRNA-targeting site 509-bp downstream of the ATG as used in the previous study (Wang et al. 2016). For this, $1 \mu \mathrm{g}$ of the Cas 9 cassette $(7 \mathrm{~kb}), 700 \mathrm{ng}$ of the sgRNA cassette ( $\sim 400 \mathrm{bp})$, and $2 \mu \mathrm{g}$ of the gene deletion 
Table 1 Deletion of ADE2 with a 1-kb arm deletion construct

\begin{tabular}{|c|c|c|c|c|c|c|}
\hline Strain & Deletion construct & Cas9 & sgRNA & Red colonies & Total colonies & $A D E 2$ disruption frequency $(\%)$ \\
\hline H99 & + & + & + & 4392 & 4728 & 92.90 \\
\hline H99 & + & + & + & 2648 & 2864 & 92.40 \\
\hline H99 & + & - & - & 20 & 1120 & 1.78 \\
\hline H99 & + & - & - & 16 & 656 & 2.44 \\
\hline cku80s H99 & + & + & + & 2080 & 2528 & 82.30 \\
\hline cku80s H99 & + & + & + & 2504 & 3184 & 78.60 \\
\hline cku80s H99 & + & - & - & 26 & 1456 & 1.78 \\
\hline cku80د H99 & + & - & - & 23 & 1888 & 1.22 \\
\hline JEC21 & + & + & + & 256 & 456 & 56 \\
\hline JEC21 & + & + & + & 512 & 760 & 67.30 \\
\hline JEC21 & + & - & - & 8 & 240 & 3.33 \\
\hline JEC21 & + & - & - & 12 & 264 & 4.55 \\
\hline XL280 & + & + & + & 472 & 720 & 65.60 \\
\hline XL280 & + & + & + & 480 & 640 & 75 \\
\hline
\end{tabular}

sgRNA, Single-guide RNA.

construct ( $\sim 4 \mathrm{~kb})$ were mixed with Cryptococcus cells of the indicated recipient strains (H99, cku80 $\mathrm{H} 99$, JEC21, and XL280) during electroporation. Transformation with only the gene deletion construct was included for comparison. All electroporated cells were plated and transformants were selected on YPD plates supplemented with the antibiotic clonNAT.

Hundreds to thousands of transformants from electroporation were obtained for all the strain backgrounds (600 4700 for H99, 1400 3100 for cku80 $\mathrm{H99}, 200 \sim 700$ for JEC21, and $~ 700$ for XL280) (Figure 2 and Table 1). Less than $5 \%$ of the transformants were red colonies when only the gene deletion construct was used. This was true for both serotype A and D ( $2 \%$ for $\mathrm{H} 99$ and $~ 4 \%$ for JEC21) (Table 1 ). Amazingly, when combined with the CRISPR-Cas9 system, the proportion of red colonies generated by electroporation drastically increased (Figure 2 and Table 1). Greater than $90 \%$ of the transformants for $\mathrm{H} 99, \sim 80 \%$ for cku $80 \Delta \mathrm{H} 99,>50 \%$ for JEC 21 , and $>65 \%$ for XL280 were red colonies, indicating a high frequency of $A D E 2$ disruption among the transformants. Twenty red colonies from the transformants in the H99 background were randomly picked and tested for stability by five consecutive passages on nonselective medium. Nineteen of them remained NATresistant after five passages, indicating stable integration of the deletion construct into the genome of the red colonies.

To our surprise, the deletion of CKU80, which impairs NHEJ, did not show much effect on the proportion of red colonies compared to that of the wild-type H99 with or without the CRISPRCas9 system. When only the gene deletion construct was used, $1.5 \%$ of transformants in cku $80 \Delta \mathrm{H} 99$ vs. $2.11 \%$ of the transformants in H99 were red colonies. This observation is consistently with the idea that the nonintegration events are dominant during electroporation, which likely masks the difference between the two minor integration events (NHEJ and HR). Unexpectedly, when the CRISPR-Cas9 system was used together with the gene deletion construct, $\sim 80 \%$ of transformants in cku80 $\mathrm{H} 99$ vs. $92 \%$ of the transformants in $\mathrm{H} 99$ were red colonies. The lack of dramatic difference between wild-type and the NHEJ mutant could be due to the extremely high efficiency of the site-specific targeting and integration by the CRISPR-Cas9 system itself.
Although accumulation of the red pigment in the transformants indicates the disruption of Ade2's function, this disruption could be caused by either indels (insertion/deletions) after nonhomologous DNA repair, insertion of the construct into the $A D E 2$ gene, or homologous replacement using the gene knockout construct as the donor. To distinguish these possibilities, we examined the $A D E 2$ locus in 10-12 randomly selected red colonies from transformants in H99 using diagnostic RFLP. Nine out of 10 candidates in the H99 background showed the RFLP pattern indicative of correct gene deletion via homologous replacement in these mutants (Figure 3, A and D). Similarly, we examined 12 transformants in the XL280 background by diagnostic PCR as the correct mutant should yield a band of $\sim 4.4 \mathrm{~kb}$. Five out of 12 showed the expected band size indicative of correct integration (Figure 3C). The results indicate that this TRACE system can effectively generate single-gene deletion mutants through HR.

Of the three DNA fragments used in transformation in the TRACE system, only the gene knockout construct carried a selective marker (left panel in Figure 1). Based on the high percentage of red colonies generated by the TRACE system compared to the conventional method where only the gene knockout construct was used in transformation, the majority of the transformants obtained from the TRACE system likely took in all three DNA fragments during electroporation. As neither the Cas9 construct nor the sgRNA construct contained any selection marker, we postulate that not all transformants would maintain these constructs if these elements were not integrated into the genome. To test this hypothesis, we examined the presence of CAS9 in randomly selected red colonies. None of the 10 examined red colonies from the H99 background yielded the band $(\sim 4.4 \mathrm{~kb})$ indicative of the presence of the CAS9 ORF [Figure 3, A(ii) and C]. Seven out of 12 examined red colonies from the XL280 background showed no amplification of the CAS9 ORF (Figure S1B in File S1). Thus, most or all of the red colonies tested did not retain CAS9 in their genome. Diagnostic PCR was also used for testing the existence of sgRNA (Figure S1A in File S1). All 10 candidates examined in the H99 background showed a 


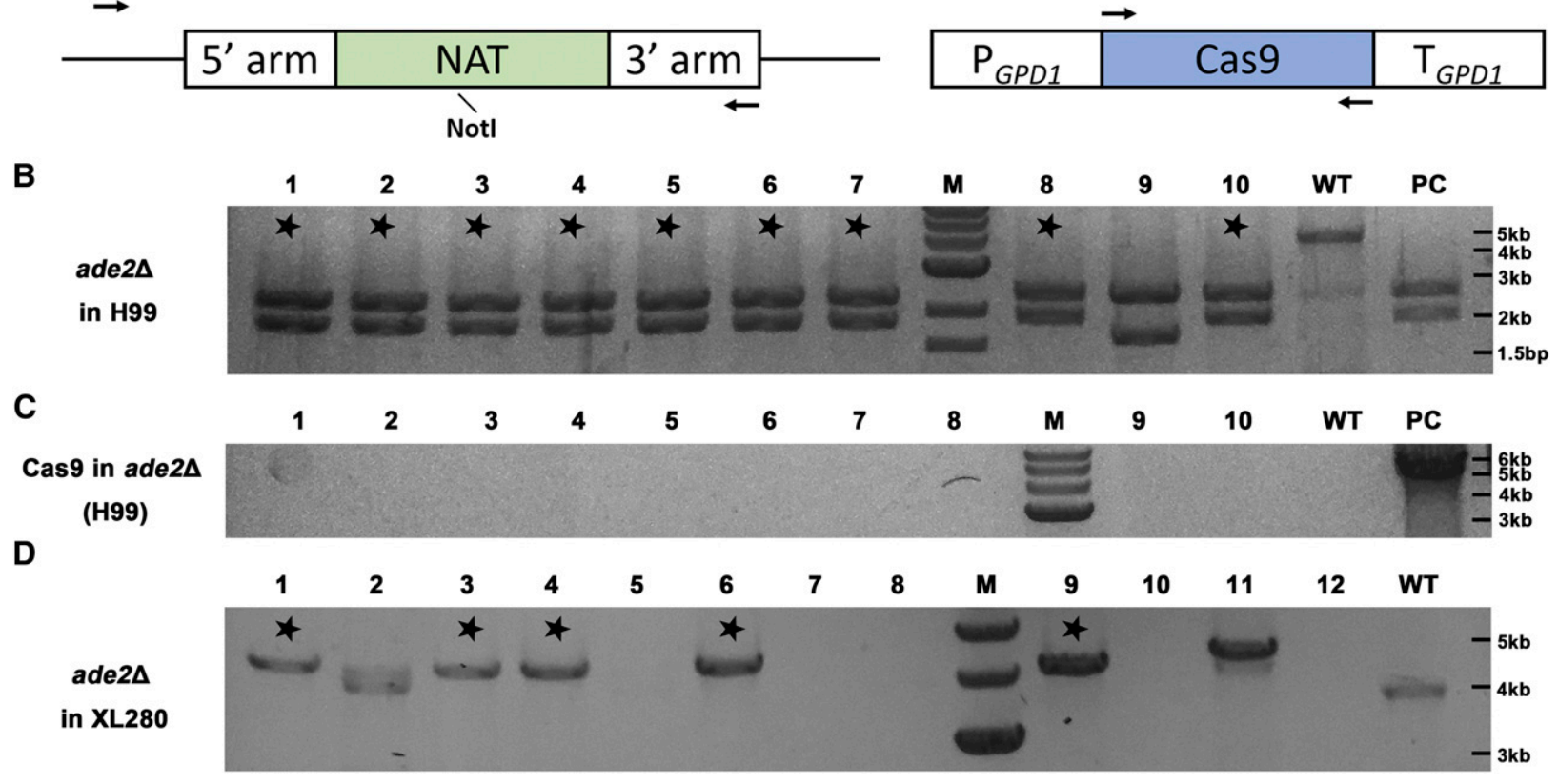

Figure 3 Deletion of ADE2 (1 kb arms) using TRACE [Transient CRISPR (clustered regularly interspaced short palindromic repeat)-Cas9 coupled with Electroporation] showed transient CAS9 expression and high efficiency of homologous integration in both serotype A and D strain backgrounds. (A) A diagram for the diagnostic RFLP for correct homologous replacement of the ADE2 gene (i). Arrows indicate the positions of the primers for PCR. For candidates in the $\mathrm{H} 99$ background, PCR product was digested by the restriction enzyme Notl. A diagram of Cas 9 with arrows pointing to the positions of the primers used to amplify the Cas9 coding sequence (ii). (B) RFLP analysis of randomly selected ade2 $\Delta$ candidates in the H99 background. Candidates that showed correct bands $(2.3$ and $1.8 \mathrm{~kb}$ ) after Notl digestion were marked with stars. Wild-type (WT) (H99) showed one single $4.2-\mathrm{kb}$ band because it lacks a Notl cutting site. Genomic DNA of an ade2s strain from our previous study (YP27) was used as a positive control (PC). M, marker. (C) No Cas9 coding sequence $(4.4 \mathrm{~kb})$ could be detected in any of the selected ade2 $\Delta$ candidates (H99) or the WT strain. Plasmid pDD162 was used as a PC. (D) In the PCR analysis of ade2 $\Delta$ candidates in the XL280 background, candidates that showed the correct band (4.4 kb) were marked with stars. WT showed one single 3.6-kb band. NAT: nourseothricin resistance cassette

faint band (Figure S1C in File S1), suggesting that sgRNA might be integrated. This issue will be addressed later.

\section{Shortened homologous arms can still generate correct HR}

In fungal species where homologous replacement is infrequent, like C. neoformans and A. fumigatus, relatively long homologous flanking sequences are needed for targeted mutagenesis when CRISPR-Cas9 is not used in transformation. Accordingly, 1-kb homologous arms are often used in Cryptococcus and our results shown above indicate that arms of this length are sufficient in our TRACE system to generate gene deletion mutants. As shorter homologous arms will make it easier to generate the gene deletion construct by double-joint PCR, we decided to test the efficiency of using shorter arms in our TRACE system. First, we reduced the length of the homologous arms of the ADE2 deletion construct to $500 \mathrm{bp}$. We used the same system to transform $\mathrm{H} 99$ as indicated in Figure 1 and Figure 2. One-fifth of electroporated cells were plated and yielded 294 colonies. Of these, 222 showed a red color suggesting that the $A D E 2$ disruption efficiency is $\sim 75 \%$. We again examined 12 randomly picked red colonies for the correct gene replacement by RFLP (Figure 4A). Eleven out of 12 candidates showed correct replacement of the ADE2 gene with the NAT drug marker. None of them were positive for the presence of
CAS9 based on diagnostic PCR (Figure S2A in File S1). This result demonstrated that a homologous arm of $500 \mathrm{bp}$ can still efficiently generate correct gene deletion mutants through HR using our TRACE system.

\section{Extremely short homologous arms (50 bp) are not sufficient for homologous gene replacement}

In Saccharomyces cerevisiae, genomic integration and HR of introduced DNA are highly efficient. This feature makes this model yeast a good system to manipulate genetically as homologous arms can be directly included in the designed primers (Rothstein 1983), consequently eliminating the need for double-joint PCR to generate gene deletion constructs. In A. fumigatus, microhomology arms as short as $35-50 \mathrm{bp}$ are sufficient for manipulating the genome when coupled with the CRISPR-Cas9 system (Zhang et al. 2016; Al Abdallah et al. 2017). To see if we could further shorten the homologous arms for gene deletion in Cryptococcus, we decided to test the efficiency of the $A D E 2$ gene deletion construct with homologous arms of $50 \mathrm{bp}$. Thus, 50-bp sequences for the homologous arms were directly included in the primers and the $A D E 2$ gene deletion construct was made by a single round of regular PCR. We then transformed H99 using the TRACE system as described earlier. We plated one-fifth of the electroporated cells and obtained 230 transformants. Of these, 


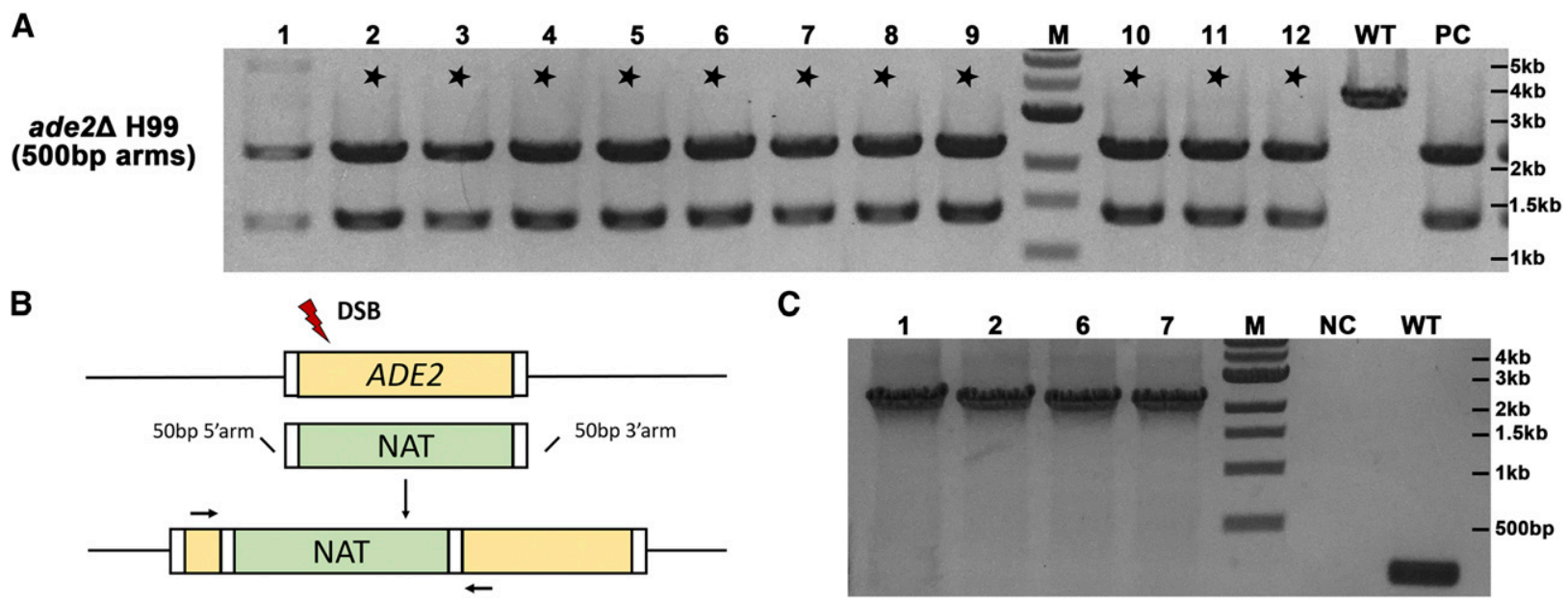

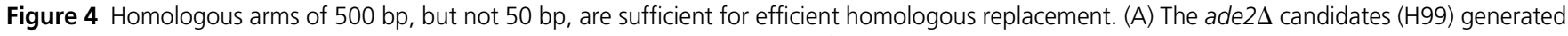
with the deletion construct carrying 500-bp homologous arms were screened for homologous replacement by RFLP analysis. Candidates with correct 2.3- and 1.8-kb bands after Notl digestion were marked with stars. Wild-type (WT) (H99) showed one single 4.2-kb band. Genomic DNA of a previously confirmed ade2 $\Delta$ mutant was used as a positive control (PC). M, marker. (B) A diagram for the insertion rather than replacement of the ADE2 locus with the construct carrying 50-bp arms. Positions of the primers used for the PCR screen in (C) are indicated with arrows. (C) PCR screen of transformants generated with the deletion construct with 50-bp arms. Candidates with insertion should yield a band of $2.1 \mathrm{~kb}$ while WT should yield a band of $\sim 200$ bp. The negative control (NC) had no DNA template. NAT: nourseothricin resistance cassette

146 were red, indicating a $60 \%$ disruption rate of $A D E 2$. To determine how many of the disruption events were caused by the replacement of the $A D E 2$ gene with the gene deletion construct, we randomly selected eight red colonies and performed diagnostic PCR as depicted in Figure 3A(i). None of the examined candidates showed the $4.2 \mathrm{~kb}$ band indicative of the $A D E 2$ gene replacement that we observed earlier using the knockout constructs with longer homologous arms (Figure 3B). The majority of them showed a larger band ( $\sim 6 \mathrm{~kb})$ (Figure S2B in File S1), which suggested that the knockout construct was possibly inserted into the DSB site within the $A D E 2$ gene rather than replacing the $A D E 2$ gene. To test if the $A D E 2$ gene was indeed disrupted by insertion rather than replacement in these red colonies, we designed a set of primers inside of the $A D E 2$ gene for screening (Figure $4 \mathrm{~B}$ ). The wild-type strain should give a band $\sim 200 \mathrm{bp}$. If the $A D E 2$ gene was replaced by the drug marker, no product should be amplified. If the knockout construct was inserted into the DSB sites within the ADE2 gene, a product of $2.1 \mathrm{~kb}$ should be amplified. The wild-type strain $\mathrm{H} 99$ and one ade2s were included as controls. All of the four candidates tested yielded a product of $2.1 \mathrm{~kb}$, consistent with insertion rather than replacement (Figure 4C). The result suggests that 50-bp arms are not sufficient for HR in generating gene deletion in Cryptococcus. Although this approach does not create a clean replacement of the targeted gene, it could still be used to disrupt a gene's function by insertion.

Precise insertion of an introduced DNA fragment at the DSB site could be useful in other applications, such as tagging proteins at their native loci. To examine if additional changes such as indels occurred when this NAT construct was inserted into the ADE2 DSB site, we sequenced the four candidates. Since the construct may get inserted in two different orien- tations, the sequence from each candidate was aligned with two expected sequences. Two primers amplifying from either end were used for each candidate to get a full-length sequence. Three of them carried the insertion in one direction (Figure S4A-i in File S1) while the other one was inserted in the opposite direction (Figure S4A-ii in File S1). Among these four candidates, isolate 7 showed precise insertion without any indels (Figure 4A). Isolate 2 also showed precise insertion at the junctions. The results suggest that NHEJ repair may contribute to DSB repair and can generate precise insertion.

\section{Transformation efficiency and gene disruption rate depends on the dose of Cas9 and sgRNA}

To determine if the efficiency of the TRACE system depends on the concentration of Cas9 and sgRNA used, we repeated the experiment to delete the ADE2 gene in H99 cells with lower doses of CRISPR-Cas9 elements. We tested three different combinations of Cas9/sgRNA ( $1 \mu \mathrm{g} / 700 \mathrm{ng}, 0.5 \mu \mathrm{g} / 350 \mathrm{ng}$, and $0.2 \mu \mathrm{g} / 150 \mathrm{ng}$ ). The $A D E 2$ gene deletion construct with the 1-kb arm was kept at the same concentration $(2 \mu \mathrm{g})$ and the cell concentration for transformation was also kept the same. We again plated one-fifth of the transformed cells, and found that $90.5 \%$ of the 351 colonies with the high dose, $69.9 \%$ of the 318 colonies with the medium dose, and $40.4 \%$ of the 104 colonies with the low dose were red (Figure 5 and Table 2). This result indicates that the rate of gene disruption positively correlates with the dose of Cas9/sgRNA when the deletion construct remains the same. This result also shows that lowering the dose of Cas9/sgRNA to as low as $0.2 \mu \mathrm{g} /$ $150 \mathrm{ng}$ is sufficient for the generation of ade $2 \Delta$ mutants. We noticed that the number of transformants on selective medium dropped when the dose of CRISPR-Cas9 elements was 


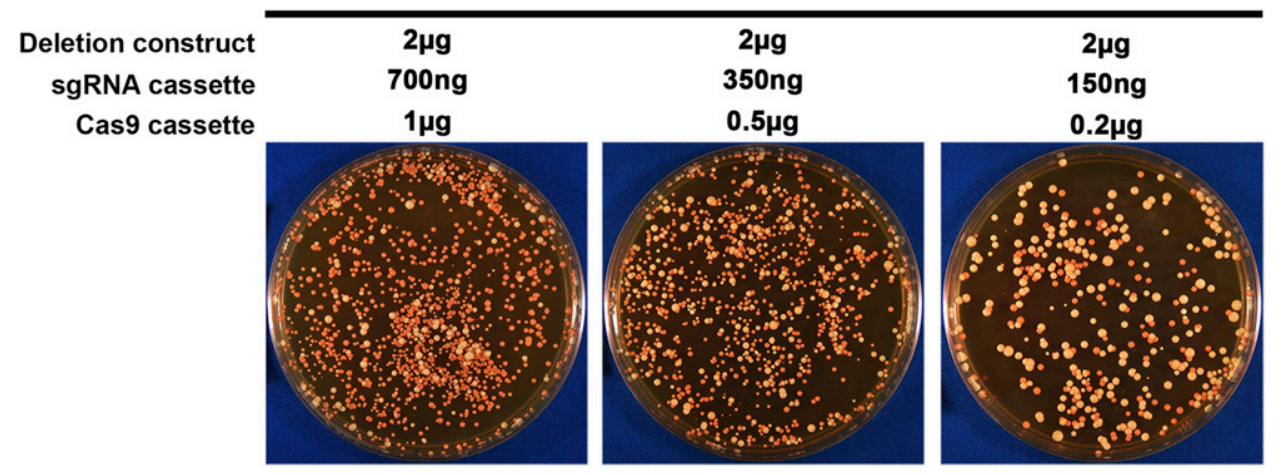

Figure 5 Transformation efficiency and gene disruption rate are dependent on the dose of the CRISPR (clustered regularly interspaced short palindromic repeat)-Cas9 elements. Different doses of Cas9 and single-guide RNA (sgRNA) were used to transform the same batch of H99 cells using TRACE (Transient CRISPR-Cas9 coupled with Electroporation).

reduced. This suggests that the transformation efficiency is also affected by the concentration of Cas9/sgRNA.

When we further reduced the dose of Cas9/sgRNA to 170/ $100 \mathrm{ng}$, the proportion of red colonies among transformants was further reduced (Figure 6A). However, compared to the transformation without Cas9/sgRNA, the proportion of red mutants was still much higher. This was true for both the serotype A strain H99 (15.85\% vs. 2.70\%) and the serotype D strain XL280 (45.35\% vs. 0.39\%) (Table 3) (Figure 6A). Interestingly, we did not see any apparent difference in the percentage of red colonies among the transformants between the wild-type $\mathrm{H} 99$ and the cku80 $\Delta$ mutant. This again is consistent with our earlier observation (Figure 2), and suggests that DSBs created by Cas9/sgRNA are critical and sufficient to promote integration and homologous replacement when appropriate donor DNA is present.

Given that most transformants generated from conventional electroporation are unstable because of the predominance of nonintegration events of introduced DNA, we decided to examine the stability of transformants generated by TRACE with the low dose of Cas9/sgRNA. We randomly picked 25 red colonies and 25 white colonies from the transformants in the H99 background. After five consecutive passages on nonselective YPD medium, cells were replicated onto YPD medium and also onto the selective medium supplemented with NAT. Twenty-four out of 25 red colonies were stable, whereas only 4 out of 25 white colonies were stable (Figure 6B). This indicates that red colonies of potential ade2 mutants are stable. We further picked 50 white colonies generated by the TRACE system and 50 white colonies generated by normal electroporation and tested their stability as described earlier. Again, similarly low numbers (22 and 24\%, respectively) of stable candidates were obtained (Figure 6C), corroborating the low frequency of integration when DNA was introduced by electroporation.
We further tested the red transformants generated with the low dose of Cas9/sgRNA for the replacement of the $A D E 2$ gene and for the presence/absence of the CRISPR-Cas9 elements. Seven out of 12 randomly selected H99 transformants showed correct gene deletion (Figure 6D). As mentioned earlier, sgRNA could integrate into the cryptococcal genome in some of the transformants when the high dose of Cas9/sgRNA was used (Figure S1 in File S1). To test if such unwanted integration events were reduced among the transformants obtained from experiments with a reduced dose of sgRNA, we tested the presence of sgRNA by PCR of these 12 candidates. We could not detect sgRNA in five of the transformants and two transformants showed very faint bands (Figure 6E). No CAS9 was detected in any of the 12 transformants (Figure $6 \mathrm{~F}$ ). Thus, reducing the dose of Cas9/sgRNA allows the generation of correct gene deletion mutants without integration of either CAS9 or sgRNA.

Although it is beyond the scope of this current study, it is important to point out that additional measures should be taken to verify that the phenotypes observed in the selected transformants are caused by the desired mutation. For mutants generated by TRACE, mutants should be screened for the absence of the CRISPR-Cas9 elements. In cases where mutants with the presence of CAS9 or sgRNA have to be used, crossing the mutant with a wild-type partner should help obtain clean mutants if these elements are not linked to the desired mutation. More importantly, genetic crosses in C. neoformans allow genetic linkage analyses that can verify the association of the phenotype with the intended genotype. Additionally, gene complementation can verify the causative effect of the gene disruption, as we demonstrate later in this study.

\section{Multiple gene deletions can be achieved in one transformation by the TRACE system}

In diploid organisms, deletion of a gene usually requires consecutive transformations to replace both alleles. The CRISPR-Cas9

Table 2 Gene disruption rate is dose-dependent

\begin{tabular}{lcccccc}
\hline Strain & Deletion construct $(\mu \mathrm{g})$ & Cas9 $(\mu \mathrm{g})$ & sgRNA $(\mathrm{ng})$ & Red colonies & Total colonies & ADE2 disruption frequency $(\%)$ \\
\hline H99 & 2 & 1 & 700 & 318 & 351 & 90.6 \\
H99 & 2 & 0.5 & 350 & 221 & 316 & 69.9 \\
H99 & 2 & 0.2 & 150 & 42 & 104 & 40.4 \\
\hline
\end{tabular}

sgRNA, Single-guide RNA. 
A

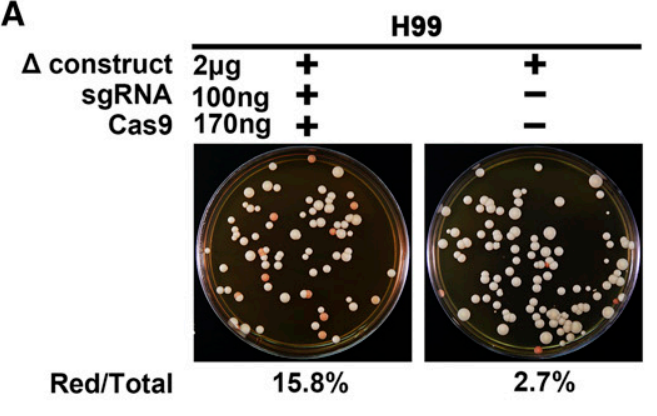

B
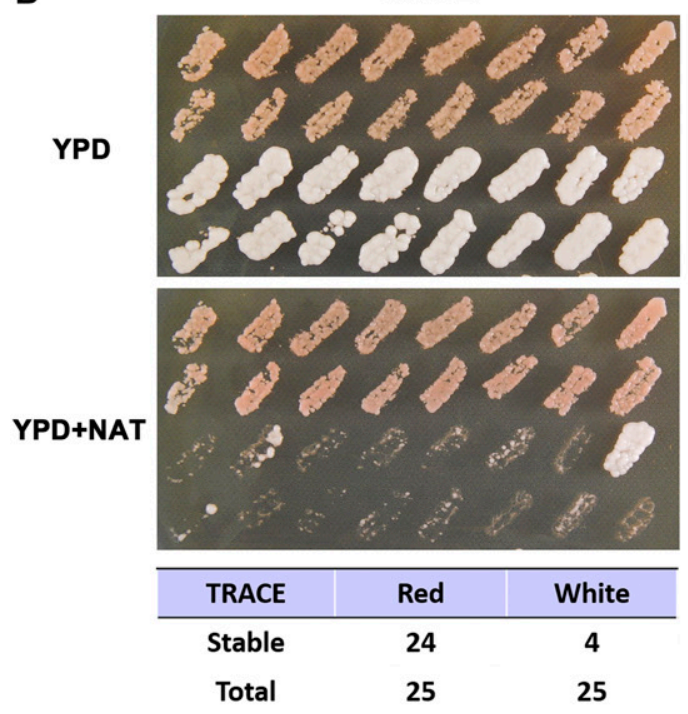

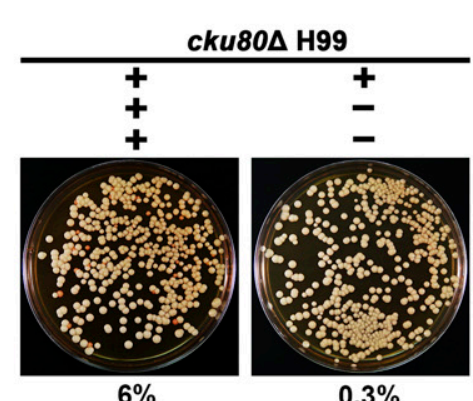

$6 \%$

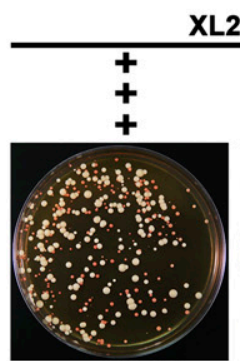

$45.35 \%$
XL280

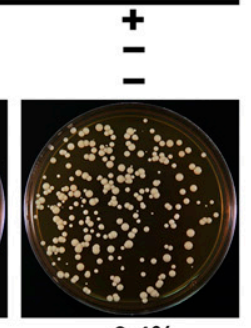

$0.4 \%$

TRACE C

C TRACE

YPD

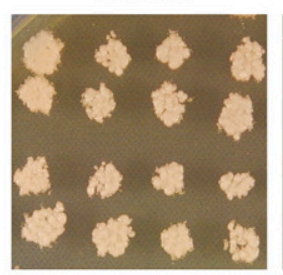

Electroporation
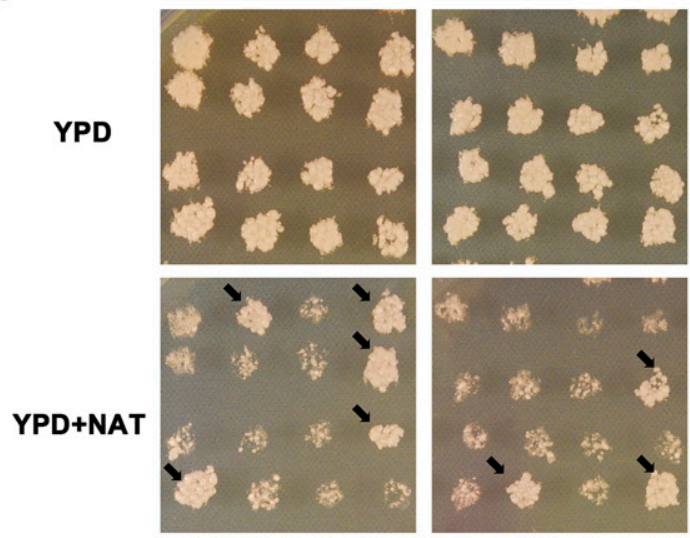

\begin{tabular}{ccc}
\hline White & TRACE & Electroporation \\
\hline Stable & 11 & 12 \\
Total & 50 & 50 \\
\hline
\end{tabular}

D

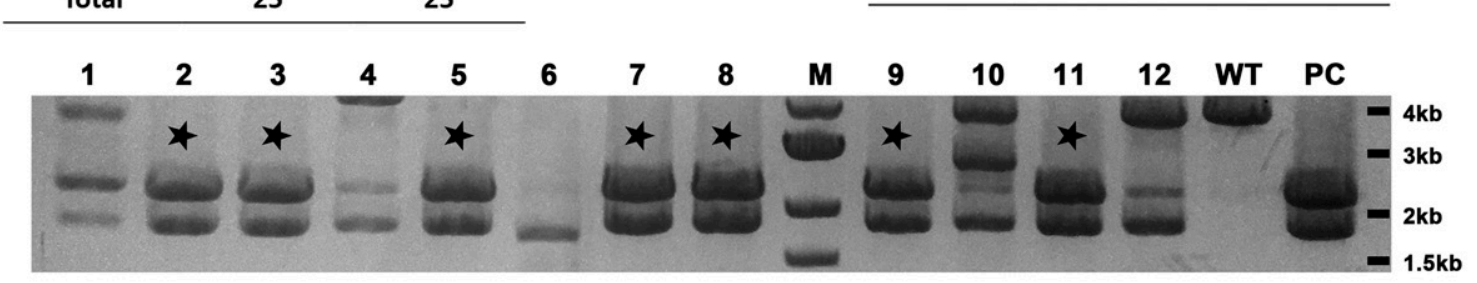

E

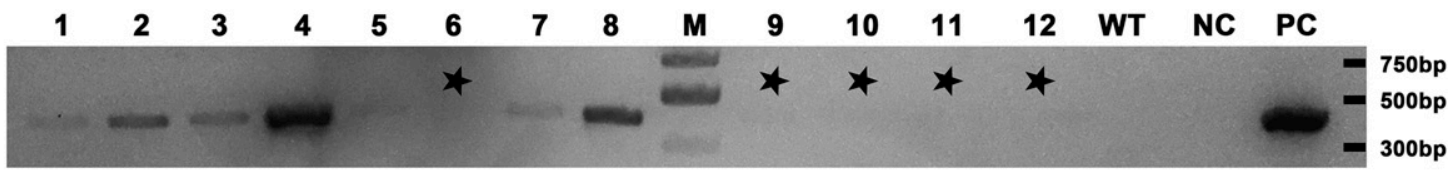

F

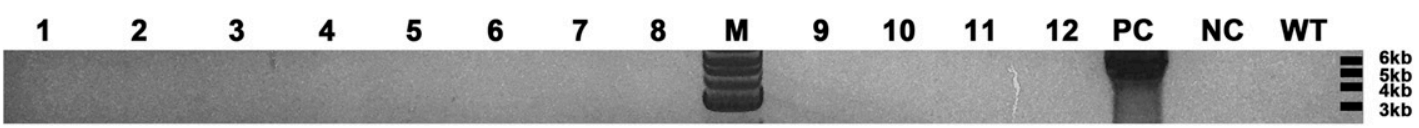

Figure 6 Low concentration of clustered regularly interspaced short palindromic repeat (CRISPR)-Cas9 elements are sufficient for correct gene deletion and can reduce the chance of single-guide RNA (sgRNA) integration. (A) Transformation of serotype A and serotype D strains with TRACE (Transient CRISPR-Cas9 coupled with Electroporation) (100 ng sgRNA and $170 \mathrm{ng}$ Cas9) or without TRACE to disrupt the ADE2 gene. (B) After five consecutive passages on nonselective YPD medium, red and white colonies generated by TRACE were replicated onto nonselective and selective medium. Nonstable transformants showed spotty growth on selective medium. (C) White colonies generated from TRACE and normal electroporation were tested for stability after five passages. Black arrows indicated stable candidates. (D) The ade2 $\Delta$ candidates generated by TRACE with the low dose of sgRNA and Cas 9 were screened for homologous replacement by RFLP. Candidates that yielded correct bands of 2.3 and $1.8 \mathrm{~kb}$ after Notl digestion were marked with stars. Wild-type (WT) (H99) showed one single 4.2-kb band. Genomic DNA of one ade2 $\Delta$ strain served as the positive control (PC). M, marker. (E and F) The ade $2 \Delta$ candidates generated by TRACE with the low dose of sgRNA and Cas9 were screened for the presence of sgRNA (E) and CAS9 (F). The negative control (NC) had no DNA template.

system is a powerful tool because it can recognize both alleles of the same gene and consequently a homozygous deletion mutant can be made in one transformation. This property made the
CRISPR-Cas9 transformation such a popular method shortly after it was introduced to the diploid fungus C. albicans. Cryptococcus, like most other fungal species, mostly exists in a haploid state. 
Table 3 Transformation efficiency of ADE2 deletion construct with 1-kb arms

\begin{tabular}{|c|c|c|c|c|c|c|}
\hline Strain & Deletion construct & Cas9 & sgRNA & Red colonies & Total colonies & $A D E 2$ disruption frequency (\%) \\
\hline H99 & + & + & + & 13 & 82 & 15.85 \\
\hline H99 & + & - & - & 3 & 111 & 2.70 \\
\hline cku80د H99 & + & + & + & 29 & 489 & 5.93 \\
\hline cku80s H99 & + & - & - & 2 & 548 & 0.36 \\
\hline XL280 & + & + & + & 161 & 355 & 45.35 \\
\hline XL280 & + & - & - & 1 & 258 & 0.39 \\
\hline
\end{tabular}

sgRNA, Single-guide RNA.

However, there are duplicated genes in the Cryptococcus genome (Fraser et al. 2005) and there are also conserved gene families. Here, we decided to use the pheromone gene family in C. neoformans to determine if our TRACE system could be used to delete multiple genes in one transformation.

Cryptococcal pheromone is a peptide signal that initiates recognition between the compatible mating partners and subsequent cell-cell fusion. Pheromone is essential for cryptococcal $\alpha-\mathbf{a}$ bisexual mating and the production of mating hyphae (Shen et al. 2002; Lin and Heitman 2006; Lin et al. 2010) (Figure 7D). In H99 cells (serotype A, mating type $\alpha$ ) there are four pheromone genes, $M F \alpha 1-4$, located in the mating type locus (Lengeler et al. 2002) (Figure 7A). $M F \alpha 1$ and $M F \alpha 2$ genes are located next to each other, and $M F \alpha 3$ and $M F \alpha 4$ are neighboring genes (Figure 7A). The ORF sequences of the $M F \alpha 2,3$, and 4 genes are identical, and they differ only slightly from that of $M F \alpha 1$ (Figure 7C).

We first decided to test the possibility of generating double $m f \alpha 1 \Delta 2 \Delta$ mutants in one transformation without nonspecific disruption of the similar $M F \alpha 3$ and $M F \alpha 4$ locus nearby. We designed one sgRNA that specifically targeted the 3 ' sequence of $M F \alpha 1$ (blue sequence in Figure 7C) but not the other three pheromone genes, and generated a deletion construct with the NAT drug resistance marker carrying the homologous arms for the $M F \alpha 1-2$ locus (Figure 7B). Unlike the ade 2 mutants, where red colonies are mostly stable and can be easily identified visually, the $m f \alpha 1 \Delta 2 \Delta$ mutants are not visually different from the wild-type. To examine the efficiency of DNA integration with the TRACE approach, we randomly picked 32 transformants for stability testing and found that 30 of the tested isolates were stable. This observation suggests that the vast majority of the transformants had the knockout construct integrated into the genome. Eight stable isolates were then streaked for single colonies and examined for correct replacement of the $M F \alpha 1-2$ genes by diagnostic PCR. All of the eight colonies examined showed the expected $\sim 4-\mathrm{kb}$ band in PCR confirmation of correct integration and replacement of $M F \alpha 1-2$ (Figure S3A in File S1). The $m f \alpha 1 \Delta 2 \Delta$ mutants successfully mated with a wild-type partner and generated mating filaments on mating-inducing V8 juice agar medium (Figure 7E), indicating that the other two pheromone genes remained functional in the tested strains. This result suggests that the designed sgRNA for $M F \alpha 1$ was highly specific and did not target the other homologous $M F \alpha$ genes in the nearby genetic locus at a noticeable frequency.
We next decided to test the possibility of generating quadruple $m f \alpha 1 \Delta 2 \Delta 3 \Delta 4 \Delta$ mutants in one transformation. To this aim, we designed one sgRNA that matched the conserved sequence of $M F \alpha 1-4$ (red asterisks in Figure 7B red sequence in Figure 7C) and two deletion constructs as shown in Figure $7 \mathrm{~B}$; one deletion construct with the NAT drug resistance marker carrying the homologous arms for the $M F \alpha 1-2$ locus and the other deletion construct with the NEO drug resistance marker carrying the homologous arms for the $M F \alpha 3$ 4 locus. Four DNA fragments, Cas9 $(1 \mu \mathrm{g})$, sgRNA (700 ng), and the two deletion constructs (1.2 $\mu \mathrm{g}$ each) were mixed with $\mathrm{H} 99$ cells during electroporation. After transformation, cells were first plated onto agar medium with a single selective drug (YPD + NAT or YPD + G418) and then replicated onto YPD agar with both selective drugs (YPD + NAT + G418). About $38 \%$ of the transformants selected on the NAT plates were also resistant to G418. Conversely, 55\% of the transformants selected on the G418 plates were also resistant to NAT. The result indicates that incorporation of both constructs occurred at an appreciably high frequency among the transformants.

For those double drug-resistant mutants, we tested the integration of two deletion constructs by diagnostic PCR (Figure S3, B and C). The PCR results indicate that 6 candidates out of 12 examined contained both correctly integrated deletion constructs. Consistently, all six mutants tested for phenotype failed to mate with a wild-type a partner and the coculture did not produce any mating hyphae, in contrast to the wild-type H99 parental strain (Figure 7E). The phenotype of the $m f \alpha 1 \Delta 2 \Delta 3 \Delta 4 \Delta$ mutants was similar to the known mat2 $\Delta$ mutant (Figure 7E), where the transcription factor Mat2 of the pheromone pathway was disrupted (Lin et al. 2010; Wang and Lin 2011; Feretzaki and Heitman 2013; Gyawali et al. 2017). After prolonged incubation, few rudimentary hyphal sprouts could occasionally be observed in the $m f \alpha 1 \Delta 2 \Delta 3 \Delta 4 \Delta$ mutants, likely due to poor pheromoneindependent filamentation under this condition as we also observed in the mat2 $\Delta$ mutant (Gyawali et al. 2017) (Figure $7 \mathrm{E}$ ). Collectively, our results indicate that one single sgRNA can target multiple genes and that multiple gene deletions are achievable in a single transformation in our TRACE system.

\section{Complementation by ectopic insertion into the specific safe haven region does not need any homologous arm}

Complementation in a gene deletion mutant is critical in verifying the function of the gene deduced from the 
A

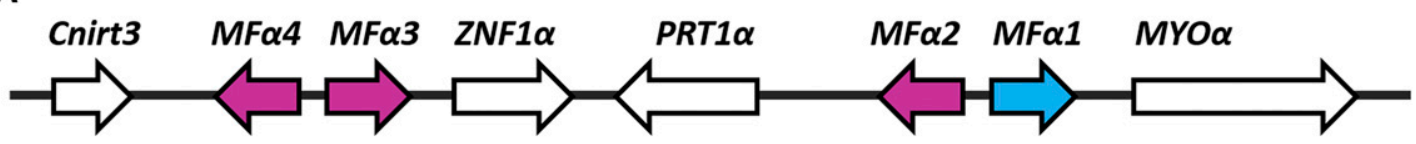

B

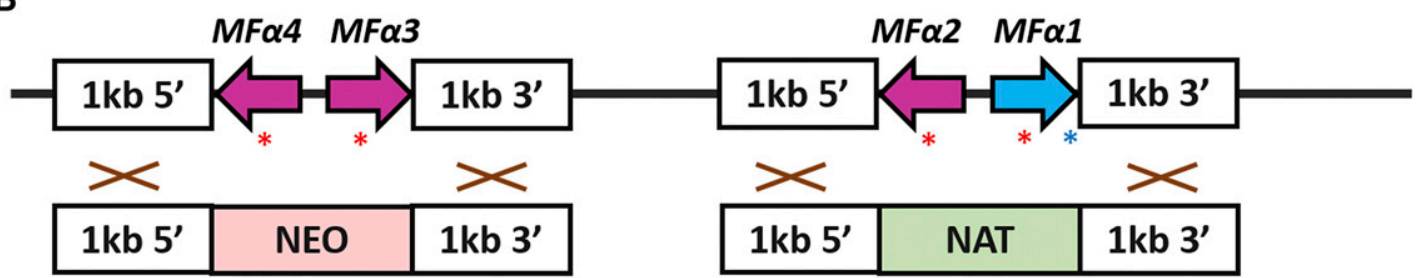

C

sgRNA for MFalpha1-4

sgRNA for MFalphal

MFalphal ATG CTCTTCTGAAGTCCCTCGCAACCAGGAGG TTTGTGTCATCGCCTAAGTTCCCAAGCAGTCAAGCCGGTGAGT MFalpha2 ATG CTCTTCTGAAGTCCCTCGCAACCAGGAGG TATGCGTCATCGCCTAAACATCCCCTCTGCGTGTTAGTCGACA MFalpha3 ATG CTCTTCTGAAGTCCCTCGCAACCAGGAGG TATGCGTCATCGCCTAAACATCCCCTCTGCGTGTTAGTCGACA MFalpha4 ATG CTCTTCTGAAGTCCCTCGCAACCAGGAGG TATGCGTCATCGCCTAAACATCCCCTCTGCGTGTTAGTCGACA

D

$\Delta / \Delta$ Pheromone

$\alpha \quad a$

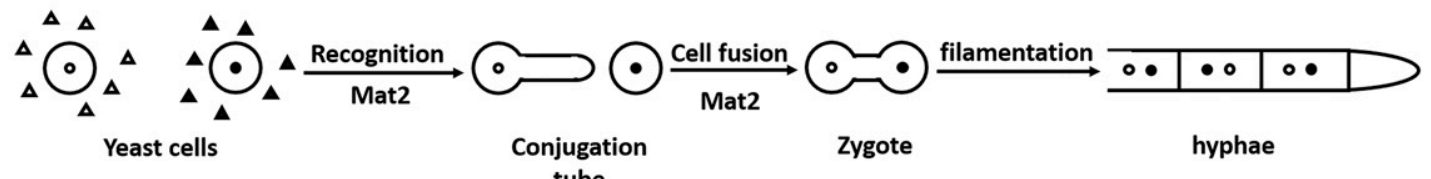

E

H99а

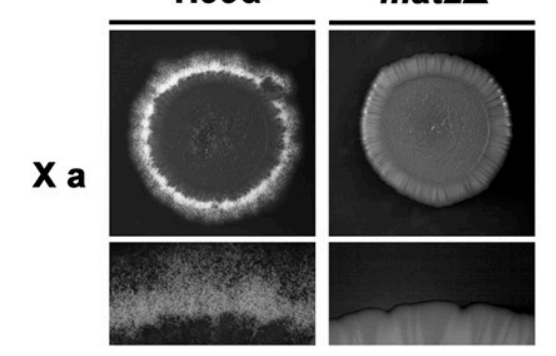

mat2ム

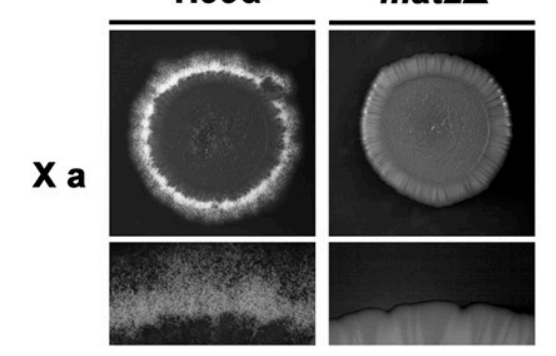
tube

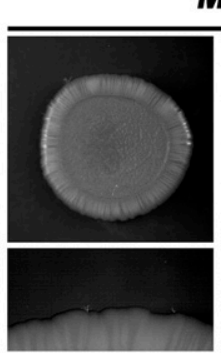

$M F \alpha 1 \Delta 2 \Delta 3 \Delta 4 \Delta$
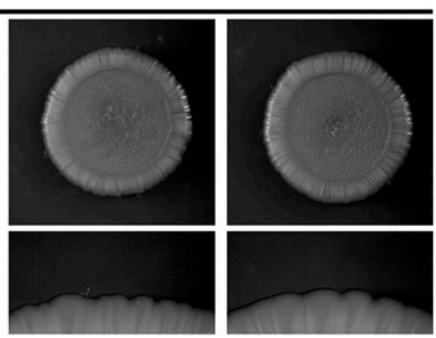

MFa1D2A

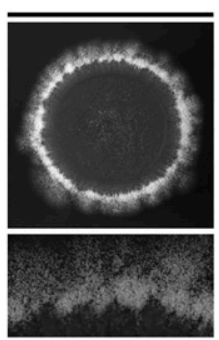

Figure 7 TRACE [Transient CRISPR (clustered regularly interspaced short palindromic repeat)-Cas9 coupled with Electroporation] can generate a quadruple $m f_{\alpha} 1 \Delta 2 \Delta 3 \Delta 4 \Delta$ mutant in $\mathrm{H} 99$ with a single transformation. (A) A diagram of the mating type locus of H99. The mating type locus contains four pheromone genes, MFo 1-4. Three pheromone genes-MFo2, MFo3, and MFo4-are identical in DNA sequence. (B) A diagram of the two deletion constructs carrying NAT and NEO drug-resistance markers (NAT: nourseothricin resistance cassette; NEO: Neomycin resistance cassette). The constructs were designed to delete MF $1-2$ or $M F \alpha 3-4$ by homologous recombination. Asterisks indicate the target sequences of single-guide RNAs (sgRNAs). (C) Sequence alignment of the four pheromone genes. The coding sequences are underlined. Red and blue sequences are the target sequences of sgRNAs. The corresponding PAM (protospacer-adjacent motif) sequences are highlighted in yellow. (D) A diagram of the cellular process of bisexual mating in C. neoformans. Cell-cell recognition and cell fusion is controlled by the pheromone pathway activated by Mat2. (E) $\alpha$ isolates of wild-type (WT) H99, mat2 $2 \Delta f_{\alpha} 1 \Delta 2 \Delta 3 \Delta 4 \Delta$ (three randomly selected transformants), and $m f_{\alpha} 1 \Delta 2 \Delta$ were cocultured with the mating type a partner JEC20. Successful mating leads to filamentation that will confer a white and fluffy appearance at the colony edge. Upper panel shows the images of the whole colonies and the lower panel shows the images of the edge of each colony.

corresponding gene deletion mutant. Although the ideal approach for complementation is integration of the wild-type allele back to its original locus, often the wild-type allele is introduced ectopically to the genome of the gene deletion mutant. Ectopic integration could be problematic as the position effect of all the complemented strains varies and the insertion could potentially disrupt other important genes, causing unwanted effects. To address this issue, two groups have identified two intergenic regions in the Cryptococcus genome in which insertion of extra DNA does not influence the expression of the neighboring genes (Arras et al. 2015; Upadhya et al. 2017). The use of the safe haven region also means that the franking sequences remain the same for the complementation of different genes. However, due to the low homologous replacement rates in Cryptococcus, only a small fraction of the transformants obtained through biolistic transformation will have the correct integration of the complementation construct.

Given the high integration rate observed above in our TRACE system, we decided to test if our system can be used 
With Arm

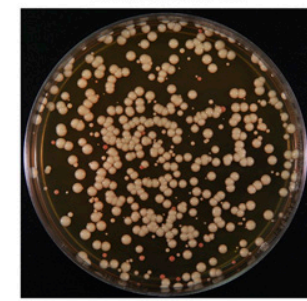

B

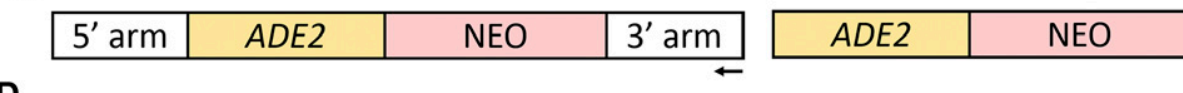

D

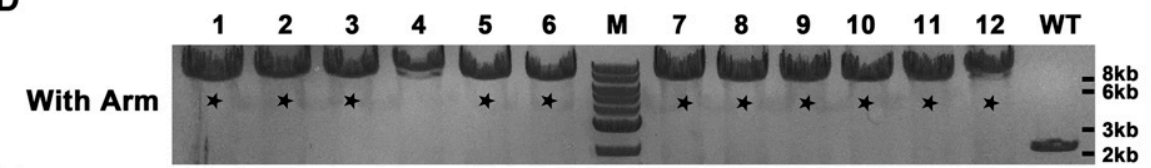

E

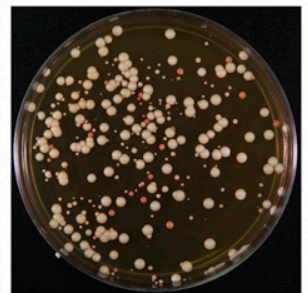

\begin{tabular}{ccc}
\hline Sample & $\begin{array}{c}\text { With } \\
\text { Arm }\end{array}$ & $\begin{array}{c}\text { W/O } \\
\text { Arm }\end{array}$ \\
\hline Red & 24 & 40 \\
White & 410 & 262 \\
\hline Efficiency & $94.5 \%$ & $86.8 \%$ \\
\hline
\end{tabular}

C

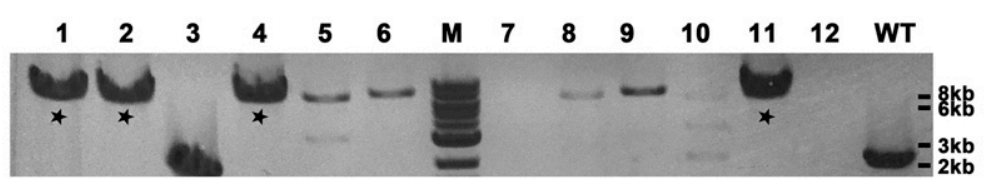

Figure $8 A D E 2$ complementation in the H99 safe haven (SH2) region by TRACE [Transient CRISPR (clustered regularly interspaced short palindromic repeat)-Cas9 coupled with Electroporation]. (A) Complementation of ade2 $\Delta$ by TRACE with the complementation construct carrying the homologous arms (left) or the complementation construct without (W/ $\mathrm{O}$ ) any homologous arms (right). (B and C) A diagram of the complementation construct with the homologous arms (B) or without arms (C) (NEO: neomycin resistance cassette). Positions of the primers used for further PCR analysis in ( $D$ and $E$ ) are indicated with arrows. ( $D$ and E) PCR screening of randomly selected white colonies from transformation with the complementation construct with the homologous arms (D) or without the homologous arms (E) for the integration events at the $\mathrm{SH} 2$ region. Correct integration into the $\mathrm{SH} 2$ region should yield a band of $7.4 \mathrm{~kb}$. Wild-type (WT) should yield a band of $2 \mathrm{~kb}$. for targeted integration at the safe haven region (SH2) (Upadhya et al. 2017). Because of the capability of precise insertion at the DSB site within the ADE2 locus that we observed earlier when very short homologous arms (50 bp) were used in our TRACE system (Figure 4, B and C), we decided to test our system for gene complementation at the $\mathrm{SH} 2$ region using two different complementation constructs; one complementation construct carries homologous arms of $1 \mathrm{~kb}$ long matching to the $\mathrm{SH} 2$ region, while the other complementation construct carries no homologous arms at all. We chose to use the ADE2 gene to complement the ade $2 \Delta$ mutants.

The first complementation construct required four DNA fragments: (i) the 1-kb long 5' SH2 homologous arm; (ii) the $A D E 2$ gene composed of its native promoter, ORF, and terminator (3.3 kb); (iii) the $N E O$ marker conferring resistance to the drug G418 (2.2 kb); and (iv) the 1-kb long 3' SH2 homologous arm (Figure 8B). These four DNA fragments along with a pUC19 vector were assembled using the NEBuilder HiFi DNA Assembly Master Mix according to the manufacturer's instructions. The second complementation construct carried no homologous arms to the $\mathrm{SH} 2$ region and thus required only the $A D E 2$ gene and the $N E O$ marker (Figure $8 C$ ). The elimination of the homologous arms greatly simplified the construction process and the construct was created by a single-joint PCR. The sgRNA was designed to target the $\mathrm{SH} 2$ region. An ade $2 \Delta$ red mutant generated earlier was used as the recipient strain. In total, $94.5 \%$ of the transformants turned white when the complementation construct carrying homologous arms was used (Figure 8A). Surprisingly, 86.8\% of the transformants turned white when the complementation construct without homologous arms was used (Figure $8 \mathrm{~A})$. The results suggest that constructs with or without the homologous arms can complement the ade $2 \Delta$ mutant efficiently. To determine if the phenotypically complemented strains from both transformations have the complementation construct integrated at the $\mathrm{SH} 2$ region, we randomly selected 12 white colonies from the experiments with the two complementation constructs (one with homologous arms and one without) and performed diagnostic PCR using the set of primers depicted in Figure 8B. Using this set of primers, the wild-type strain should yield a band of $2.1 \mathrm{~kb}$ in size, while the transformants with the complementation construct correctly inserted into the $\mathrm{SH} 2$ region should yield a band of $7.5 \mathrm{~kb}$ in size. We found that 11 out of 12 tested from the transformation with the construct carrying the homologous arms showed correct integration into the $\mathrm{SH} 2$ region, while 4 out of 12 tested from the transformation with the construct without homologous arms showed correct integration (Figure 8, D and E). Sequencing of the PCR amplicons indicated that precise insertion can occur with the gene complementation construct without arms (Figure S4B in File S1). The finding indicates that gene complementation constructs can be integrated into the $\mathrm{SH} 2$ region at a sufficiently high frequency with or without homologous arms.

\section{Discussion}

In the early 1990s, two major transformation methods, biolistic and electroporation, were developed to genetically manipulate the clinically important fungal pathogen C. neoformans (Edman and Kwon-Chung 1990; Toffaletti et al. 1993). Although biolistic transformation is currently employed as the routine approach for genetic manipulation in C. neoformans, the low transformation efficiency, variation from experiment to experiment, and high costs are discouraging. 
In this study, we introduced a simple and cost-effective transformation system, TRACE, for genetic manipulations in the C. neoformans species complex. Combining the high transformation rate of electroporation and increased $\mathrm{HR}$ rate due to CRISPR-Cas9, TRACE can generate hundreds to thousands of transformants with $\leq 90 \%$ of the gene disruption rate. It is important to note that the TRACE system relies on the successful delivery of all three independent DNA fragments (sgRNA cassette, Cas9 cassette, and donor DNA) into the recipient, which requires a highly efficient transformation method. Electroporation provides such an ideal delivery system for Cryptococcus.

One issue associated with the CRISPR-Cas9 system is potential off-target events (Fu et al. 2013; Hsu et al. 2013), which can lead to unexpected mutations in the genome. However, such risk could be minimized by carefully selecting target sequences of sgRNA and reducing the doses of Cas9 and sgRNA. Only an sgRNA with the fewest possible off-target sites should be chosen. For instance, the designed sgRNA used for $M F \alpha 1$ was highly specific and did not target the other homologous $M F \alpha$ genes in the nearby genetic locus among the transformants that we examined. Moreover, in contrast to using a recipient strain carrying integrated CRISPR-Cas9 elements, a transient system can further minimize the off-target effect as the sgRNA and Cas9 are eliminated eventually. Backcrossing the mutant to a wildtype strain is strongly recommended. This can demonstrate the genetic linkage between the phenotype and the desired mutation, as well as removing possible off-target mutations present in the original transformants. A genome sequencing approach was recently used to analyze potential secondary mutations in the genome caused by biolistic bombardment in Cryptococcus (Friedman et al. 2018). Similarly, a thorough comparison of off-target effects of a transient $v$ s. a permanent CRISPRCas9 system could be achieved by comparative genome analyses of multiple transformants.

In closing, we demonstrated that TRACE can be used to efficiently create single-gene deletion, multiple gene deletions, gene complementation, and targeted insertions. TRACE can also be used for precise knock-in for protein tagging. TRACE is not only cost-efficient; it also accelerates genetic engineering of Cryptococcus. It is conceivable that TRACE will enable laboratories in regions with limited resources for research to undertake efficient genetic manipulation of this pathogen as it only requires a PCR machine and an electroporator, two instruments commonly used in molecular biology laboratories. Given that nonintegration events and the dominance of NHEJ present a bottleneck for genetic manipulation in many other fungal pathogens (Boyce and Andrianopoulos 2015), we expect that TRACE or similar systems can be applicable in these organisms.

\section{Acknowledgments}

We thank the Lin laboratory members for their helpful suggestions and Rene Garcia for technical assistance. This work was supported by the National Institutes of Health
(http://www.niaid.nih.gov/Pages/default.aspx) (R01AI097599 to X.L.). X.L. holds an Investigator Award in the Pathogenesis of Infectious Disease from the Burroughs Wellcome Fund (http://www.bwfund.org/) (1012445 to X.L.). The funders had no role in study design, data collection and interpretation, or the decision to submit the work for publication.

Author contributions: Y.F. and X.L. conceived and designed the experiments. Y.F. performed the experiments. Y.F. and X.L. analyzed the data. X.L. contributed reagents, materials, and analysis tools. Y.F. wrote the paper. X.L. edited the paper.

\section{Literature Cited}

Al Abdallah, Q., W. Ge, and J. R. Fortwendel, 2017 A simple and universal system for gene manipulation in Aspergillus fumigatus: in vitro-assembled Cas9-guide RNA ribonucleoproteins coupled with microhomology repair templates. mSphere 2: e00446-17.

Armstrong-James, D., G. Meintjes, and G. D. Brown, 2014 A neglected epidemic: fungal infections in HIV/AIDS. Trends Microbiol. 22: 120-127. https://doi.org/10.1016/j.tim.2014.01.001

Arras, S. D., J. L. Chitty, K. L. Blake, B. L. Schulz, and J. A. Fraser, 2015 A genomic safe haven for mutant complementation in Cryptococcus neoformans. PLoS One 10: e0122916. https:// doi.org/10.1371/journal.pone.0122916

Arras, S. D., S. M. Chua, M. S. Wizrah, J. A. Faint, A. S. Yap et al., 2016 Targeted genome editing via CRISPR in the pathogen Cryptococcus neoformans. PLoS One 11: e0164322. https:// doi.org/10.1371/journal.pone.0164322

Barrangou, R., C. Fremaux, H. Deveau, M. Richards, P. Boyaval et al., 2007 CRISPR provides acquired resistance against viruses in prokaryotes. Science 315: 1709-1712. https://doi.org/10.1126/ science. 1138140

Bhaya, D., M. Davison, and R. Barrangou, 2011 CRISPR-Cas systems in bacteria and archaea: versatile small RNAs for adaptive defense and regulation. Annu. Rev. Genet. 45: 273-297. https://doi. org/10.1146/annurev-genet-110410-132430

Boyce, K. J., and A. Andrianopoulos, 2015 Fungal dimorphism: the switch from hyphae to yeast is a specialized morphogenetic adaptation allowing colonization of a host. FEMS Microbiol. Rev. 39: 797-811. https://doi.org/10.1093/femsre/fuv035

Brown, G. D., D. W. Denning, N. A. Gow, S. M. Levitz, M. G. Netea et al., 2012 Hidden killers: human fungal infections. Sci. Transl. Med. 4: 165rv13. https://doi.org/10.1126/scitranslmed.3004404

Casadevall, A., and J. R. Perfect, 1998 Cryptococcus neoformans. ASM Press, Washington, DC. https://doi.org/10.1128/ 9781555818241

Chaiwarith, R., S. Vongsanim, and K. Supparatpinyo, 2014 Cryptococcal meningitis in HIV-infected patients at Chiang Mai University Hospital: a retrospective study. Southeast Asian J. Trop. Med. Public Health 45: 636-646.

Dickinson, D. J., J. D. Ward, D. J. Reiner, and B. Goldstein, 2013 Engineering the Caenorhabditis elegans genome using Cas9-triggered homologous recombination. Nat. Methods 10: 1028-1034. https://doi.org/10.1038/nmeth.2641

Edman, J. C., 1992 Isolation of telomerelike sequences from Cryptococcus neoformans and their use in high-efficiency transformation. Mol. Cell. Biol. 12: 2777-2783. https://doi.org/10.1128/ MCB.12.6.2777

Edman, J. C., and K. J. Kwon-Chung, 1990 Isolation of the URA5 gene from Cryptococcus neoformans var. neoformans and its use as a selective marker for transformation. Mol. Cell. Biol. 10: 4538-4544. https://doi.org/10.1128/MCB.10.9.4538 
Feretzaki, M., and J. Heitman, 2013 Genetic circuits that govern bisexual and unisexual reproduction in Cryptococcus neoformans. PLoS Genet. 9: e1003688. https://doi.org/10.1371/ journal.pgen.1003688

Fox, D. S., G. M. Cox, and J. Heitman, 2003 Phospholipid-binding protein Cts1 controls septation and functions coordinately with calcineurin in Cryptococcus neoformans. Eukaryot. Cell 2: 10251035. https://doi.org/10.1128/EC.2.5.1025-1035.2003

Fraser, J. A., J. C. Huang, R. Pukkila-Worley, J. A. Alspaugh, T. G. Mitchell et al., 2005 Chromosomal translocation and segmental duplication in Cryptococcus neoformans. Eukaryot. Cell 4: 401-406. https://doi.org/10.1128/EC.4.2.401-406.2005

Friedman, R., S. R. Gish, H. Brown, L. Brier, N. Howard et al., 2018 Unintended side effects of transformation are very rare in Cryptococcus neoformans. G3 (Bethesda) . https://doi.org/ 10.1534/g3.117.300357

Fu, J., E. Hettler, and B. L. Wickes, 2006 Split marker transformation increases homologous integration frequency in Cryptococcus neoformans. Fungal Genet. Biol. 43: 200-212. https://doi.org/ 10.1016/j.fgb.2005.09.007

Fu, Y., J. A. Foden, C. Khayter, M. L. Maeder, D. Reyon et al., 2013 High-frequency off-target mutagenesis induced by CRISPRCas nucleases in human cells. Nat. Biotechnol. 31: 822-826. https:// doi.org/10.1038/nbt.2623

Fuller, K. K., S. Chen, J. J. Loros, and J. C. Dunlap, 2015 Development of the CRISPR/Cas9 system for targeted gene disruption in Aspergillus fumigatus. Eukaryot. Cell 14: 1073-1080. https://doi.org/10.1128/ EC.00107-15

Gaskell, K. M., C. Rothe, R. Gnanadurai, P. Goodson, C. Jassi et al., 2014 A prospective study of mortality from cryptococcal meningitis following treatment induction with $1200 \mathrm{mg}$ oral fluconazole in Blantyre, Malawi. PLoS One 9: e110285. https://doi. org/10.1371/journal.pone.0110285

Goins, C. L., K. J. Gerik, and J. K. Lodge, 2006 Improvements to gene deletion in the fungal pathogen Cryptococcus neoformans: absence of $\mathrm{Ku}$ proteins increases homologous recombination, and co-transformation of independent DNA molecules allows rapid complementation of deletion phenotypes. Fungal Genet. Biol. 43: 531-544. https://doi.org/10.1016/j.fgb.2006.02.007

Gyawali, R., Y. Zhao, J. Lin, Y. Fan, X. Xu et al., 2017 Pheromone independent unisexual development in Cryptococcus neoformans. PLoS Genet. 13: e1006772. https://doi.org/10.1371/journal. pgen.1006772

Haber, J. E., 2000 Partners and pathways: repairing a doublestrand break. Trends Genet. 16: 259-264. https://doi.org/10.1016/ S0168-9525(00)02022-9

Hsu, P. D., D. A. Scott, J. A. Weinstein, F. A. Ran, S. Konermann et al., 2013 DNA targeting specificity of RNA-guided Cas9 nucleases. Nat. Biotechnol. 31: 827-832. https://doi.org/10.1038/ nbt. 2647

Hsu, P. D., E. S. Lander, and F. Zhang, 2014 Development and applications of CRISPR-Cas9 for genome engineering. Cell 157: 1262-1278. https://doi.org/10.1016/j.cell.2014.05.010

Idnurm, A., and X. Lin, 2015 Rising to the challenge of multiple Cryptococcus species and the diseases they cause. Fungal Genet. Biol. 78: 1-6. https://doi.org/10.1016/j.fgb.2015.05.002

Janbon, G., K. L. Ormerod, D. Paulet, E. J. Byrnes, III, V. Yadav et al., 2014 Analysis of the genome and transcriptome of Cryptococcus neoformans var. grubii reveals complex RNA expression and microevolution leading to virulence attenuation. PLoS Genet. 10: e1004261. https://doi.org/10.1371/journal.pgen.1004261

Jinek, M., K. Chylinski, I. Fonfara, M. Hauer, J. A. Doudna et al., 2012 A programmable dual-RNA-guided DNA endonuclease in adaptive bacterial immunity. Science 337: 816-821. https:// doi.org/10.1126/science.1225829

Kim, M. S., S. Y. Kim, J. K. Yoon, Y. W. Lee, and Y. S. Bahn, 2009 An efficient gene-disruption method in Cryptococcus neoformans by double-joint PCR with NAT-split markers. Biochem. Biophys. Res. Commun. 390: 983-988. https://doi.org/10.1016/ j.bbrc.2009.10.089

Lengeler, K. B., D. S. Fox, J. A. Fraser, A. Allen, K. Forrester et al., 2002 Mating-type locus of Cryptococcus neoformans: a step in the evolution of sex chromosomes. Eukaryot. Cell 1: 704-718. https://doi.org/10.1128/EC.1.5.704-718.2002

Lin, X., and J. Heitman, 2006 The biology of the Cryptococcus neoformans species complex. Annu. Rev. Microbiol. 60: 69-105. https://doi.org/10.1146/annurev.micro.60.080805.142102

Lin, X., J. C. Jackson, M. Feretzaki, C. Xue, and J. Heitman, 2010 Transcription factors Mat2 and Znf2 operate cellular circuits orchestrating opposite- and same-sex mating in Cryptococcus neoformans. PLoS Genet. 6: e1000953. https://doi.org/10.1371/ journal.pgen.1000953

Lin, X., N. Chacko, L. Wang, and Y. Pavuluri, 2015 Generation of stable mutants and targeted gene deletion strains in Cryptococcus neoformans through electroporation. Med. Mycol. 53: 225234. https://doi.org/10.1093/mmy/myu083

Liu, O. W., C. D. Chun, E. D. Chow, C. Chen, H. D. Madhani et al., 2008 Systematic genetic analysis of virulence in the human fungal pathogen Cryptococcus neoformans. Cell 135: 174-188. https://doi.org/10.1016/j.cell.2008.07.046

Lodge, J. K., E. Jackson-Machelski, D. L. Toffaletti, J. R. Perfect, and J. I. Gordon, 1994 Targeted gene replacement demonstrates that myristoyl-CoA: protein $\mathrm{N}$-myristoyltransferase is essential for viability of Cryptococcus neoformans. Proc. Natl. Acad. Sci. USA 91: 12008-12012. https://doi.org/10.1073/pnas.91.25.12008

Mali, P., L. Yang, K. M. Esvelt, J. Aach, M. Guell et al., 2013 RNAguided human genome engineering via Cas9. Science 339: 823826. https://doi.org/10.1126/science.1232033

McDade, H. C., and G. M. Cox, 2001 A new dominant selectable marker for use in Cryptococcus neoformans. Med. Mycol. 39: 151-154. https://doi.org/10.1080/mmy.39.1.151.154

Min, K., Y. Ichikawa, C. A. Woolford, and A. P. Mitchell, 2016 Candida albicans gene deletion with a transient CRISPR-Cas9 system. mSphere 1: e00130-16.

Ni, M., M. Feretzaki, W. Li, A. Floyd-Averette, P. Mieczkowski et al., 2013 Unisexual and heterosexual meiotic reproduction generate aneuploidy and phenotypic diversity de novo in the yeast Cryptococcus neoformans. PLoS Biol. 11: e1001653. https://doi. org/10.1371/journal.pbio.1001653

Nødvig, C. S., J. B. Nielsen, M. E. Kogle, and U. H. Mortensen, 2015 A CRISPR-Cas9 system for genetic engineering of filamentous fungi. PLoS One 10: e0133085. https://doi.org/10.1371/ journal.pone.0133085

Park, B. J., K. A. Wannemuehler, B. J. Marston, N. Govender, P. G. Pappas et al., 2009 Estimation of the current global burden of cryptococcal meningitis among persons living with HIV/AIDS. AIDS 23: 525-530. https://doi.org/10.1097/QAD.0b013e328322ffac

Perfect, J. R., and T. Bicanic, 2015 Cryptococcosis diagnosis and treatment: what do we know now. Fungal Genet. Biol. 78: 4954. https://doi.org/10.1016/j.fgb.2014.10.003

Perfect, J. R., W. E. Dismukes, F. Dromer, D. L. Goldman, J. R. Graybill et al., 2010 Clinical practice guidelines for the management of cryptococcal disease: 2010 update by the Infectious Diseases Society of America. Clin. Infect. Dis. 50: 291-322. https://doi.org/10.1086/649858

Pitkin, J. W., D. G. Panaccione, and J. D. Walton, 1996 A putative cyclic peptide efflux pump encoded by the TOXA gene of the plantpathogenic fungus Cochliobolus carbonum. Microbiology 142: 1557-1565. https://doi.org/10.1099/13500872-142-6-1557

Ran, F. A., P. D. Hsu, J. Wright, V. Agarwala, D. A. Scott et al., 2013 Genome engineering using the CRISPR-Cas9 system. Nat. Protoc. 8: 2281-2308. https://doi.org/10.1038/nprot.2013.143

Rothe, C., D. J. Sloan, P. Goodson, J. Chikafa, M. Mukaka et al., 2013 A prospective longitudinal study of the clinical outcomes 
from cryptococcal meningitis following treatment induction with $800 \mathrm{mg}$ oral fluconazole in Blantyre, Malawi. PLoS One 8: e67311. https://doi.org/10.1371/journal.pone.0067311

Rothstein, R. J., 1983 One-step gene disruption in yeast. Methods Enzymol. 101: 202-211. https://doi.org/10.1016/00766879(83)01015-0

Shen, W.-C., R. C. Davidson, G. M. Cox, and J. Heitman, 2002 Pheromones stimulate mating and differentiation via paracrine and autocrine signaling in Cryptococcus neoformans. Eukaryot. Cell 1: 366-377. https://doi.org/10.1128/EC.1.3.366377.2002

Sonoda, E., H. Hochegger, A. Saberi, Y. Taniguchi, and S. Takeda, 2006 Differential usage of non-homologous end-joining and homologous recombination in double strand break repair. DNA Repair (Amst.) 5: 1021-1029. https://doi.org/10.1016/ j.dnarep.2006.05.022

Stajich, J. E., T. Harris, B. P. Brunk, J. Brestelli, S. Fischer et al., 2012 FungiDB: an integrated functional genomics database for fungi. Nucleic Acids Res. 40: D675-D681. https://doi.org/ 10.1093/nar/gkr918

Toffaletti, D. L., T. H. Rude, S. A. Johnston, D. T. Durack, and J. R. Perfect, 1993 Gene transfer in Cryptococcus neoformans by use of biolistic delivery of DNA. J. Bacteriol. 175: 1405-1411. https://doi.org/10.1128/jb.175.5.1405-1411.1993

Upadhya, R., W. C. Lam, B. T. Maybruck, M. J. Donlin, A. L. Chang et al., 2017 A fluorogenic C. neoformans reporter strain with a robust expression of $m$-cherry expressed from a safe haven site in the genome. Fungal Genet. Biol. 108: 13-25. https://doi.org/ 10.1016/j.fgb.2017.08.008

Varma, A., J. C. Edman, and K. J. Kwon-Chung, 1992 Molecular and genetic analysis of URA5 transformants of Cryptococcus neoformans. Infect. Immun. 60: 1101-1108.

Vyas, V. K., M. I. Barrasa, and G. R. Fink, 2015 A Candida albicans CRISPR system permits genetic engineering of essential genes and gene families. Sci. Adv. 1: e1500248. https://doi.org/ 10.1126/sciadv. 1500248

Walker, J. R., R. A. Corpina, and J. Goldberg, 2001 Structure of the Ku heterodimer bound to DNA and its implications for doublestrand break repair. Nature 412: 607-614. https://doi.org/ $10.1038 / 35088000$

Walton, F. J., A. Idnurm, and J. Heitman, 2005 Novel gene functions required for melanization of the human pathogen Cryptococcus neoformans. Mol. Microbiol. 57: 1381-1396. https://doi. org/10.1111/j.1365-2958.2005.04779.x

Wang, L., and X. Lin, 2011 Mechanisms of unisexual mating in Cryptococcus neoformans. Fungal Genet. Biol. 48: 651-660. https://doi.org/10.1016/j.fgb.2011.02.001

Wang, L., X. Tian, R. Gyawali, S. Upadhyay, D. Foyle et al., 2014 Morphotype transition and sexual reproduction are genetically associated in a ubiquitous environmental pathogen. PLoS Pathog. 10: e1004185. https://doi.org/10.1371/journal.ppat.1004185

Wang, Y., D. Wei, X. Zhu, J. Pan, P. Zhang et al., 2016 A 'suicide' CRISPR-Cas9 system to promote gene deletion and restoration by electroporation in Cryptococcus neoformans. Sci. Rep. 6: 31145. https://doi.org/10.1038/srep31145

Xue, C., Y.-S. Bahn, G. M. Cox, and J. Heitman, 2006 G proteincoupled receptor Gpr4 senses amino acids and activates the cAMP-PKA pathway in Cryptococcus neoformans. Mol. Biol. Cell 17: 667-679. https://doi.org/10.1091/mbc.E05-07-0699

Zhang, C., X. Meng, X. Wei, and L. Lu, 2016 Highly efficient CRISPR mutagenesis by microhomology-mediated end joining in Aspergillus fumigatus. Fungal Genet. Biol. 86: 47-57. https:// doi.org/10.1016/j.fgb.2015.12.007

Zhao, C. Z., Y. Zhang, G. L. Li, J. L. Chen, J. J. Li et al., 2015 Development of a graphical user interface for sgRNAcas9 and its application. Yi Chuan 37: 1061-1072.

Communicating editor: A. Mitchell 